\title{
On Fuchs' relation for linear differential systems
}

\author{
Eduardo Corel
}

\begin{abstract}
In this paper, we give a formal algebraic notion of exponents for linear differential systems at any singularity as eigenvalues of the residue of a regular connection on a maximal lattice (that we call 'Levelt's lattice'). This allows us to establish upper and lower bounds for the sum of these exponents for differential systems on $\mathbb{P}^{1}(\mathbb{C})$.
\end{abstract}

\section{Introduction}

Exponents are well known for homogeneous linear differential equations at a regular singularity since the classical works of Fuchs and Frobenius. Let $L \in \mathbb{C}(z)[d / d z]$ be a differential operator of order $n$ with coefficients in $\mathbb{C}(z)$. When the differential equation $L y=0$ has regular singularities over $\mathbb{P}^{1}(\mathbb{C})$, its exponents $\left(e_{i}^{s}\right)_{i=1, \ldots, n}$ for all $s \in \mathbb{P}^{1}(\mathbb{C})$ obey Fuchs' relation $[$ Poo60, ch. V, $\S 20$, p. 77]:

$$
\sum_{s \in \mathbb{P}^{1}(\mathbb{C})} \sum_{i=1}^{n}\left(e_{i}^{s}-(i-1)\right)=-n(n-1)
$$

Bertrand and Laumon (see [Ber98], also [BB85]) extended this definition in 1985 at an irregular singularity. For any linear differential equation $L y=0$, the exponents $e_{i}^{s}$ that they define satisfy the generalized Fuchs' relation

$$
\sum_{s \in \mathbb{P}^{1}(\mathbb{C})}\left(\sum_{i=1}^{n}\left(e_{i}^{s}-(i-1)\right)-\frac{1}{2} \operatorname{irr}_{s}(\text { End } \nabla)\right)=-n(n-1),
$$

where $\operatorname{irr}_{s}($ End $\nabla)$ denotes the Malgrange irregularity at $s$ of the natural connection End $\nabla$ of $\operatorname{End}_{\mathbb{C}(z)} \mathbb{C}(z)[d / d z]$ induced by the operator $L$.

In 1961, Levelt [Lev61] defined exponents for linear differential systems at a regular singular point. We extend the notion of exponents for systems at an irregular singularity (cf. Definitions 15 and 16). The main result of this paper is the following.

ThEOREM 1 (Fuchs' relation). Let $d X / d z=A X$ be a meromorphic differential system of order $n$ on $\mathbb{P}^{1}(\mathbb{C})$. The exponents $e_{1}^{s}, \ldots, e_{n}^{s}$ attached to this system at all points $s \in \mathbb{P}^{1}(\mathbb{C})$ satisfy

$$
-\frac{n(n-1)}{2} h(A) \leqslant \sum_{s \in \mathbb{P}^{1}(\mathbb{C})}\left(\sum_{i=1}^{n} e_{i}^{s}-\frac{1}{2} \operatorname{irr}_{s}(\text { End } \nabla)\right) \leqslant-h(A)+h(\operatorname{Tr} A) .
$$

The height $h(A)$ of the system is given by the formula

$$
h(A)=\sum_{s \in \mathbb{P}^{1}(\mathbb{C})} \sup \left(0,-v_{s} A d z-1\right)
$$

where $v_{s}$ is the valuation of a meromorphic function at $s \in \mathbb{P}^{1}(\mathbb{C})$ extended to $n \times n$ matrices.

Received 24 October 2001, accepted in final form 26 July 2002.

2000 Mathematics Subject Classification 34A20, 34A30, 12H05, 34C20, 32S40.

Keywords: differential system, irregular singularity, connection, Levelt lattice, exponents, Fuchs' relation.

This journal is (C) Foundation Compositio Mathematica 2004. 


\section{E. Corel}

Remark 1. The sum of exponents also satisfies

$$
\sum_{s \in \mathbb{P}^{1}(\mathbb{C})} \sum_{i=1}^{n} e_{i}^{s} \leqslant 0 .
$$

Therefore, the upper bound given in Theorem 1 is not optimal in some important cases, which we discuss in $\S 5.3$.

Remark 2. When all the singularities of the system $d X / d z=A X$ are regular, we get the relation

$$
-\frac{n(n-1)}{2} h(A) \leqslant \sum_{s \in \mathbb{P}^{1}(\mathbb{C})} \sum_{i=1}^{n} e_{i}^{s} \leqslant-h(A)
$$

that we proved in [Cor99a], as well as Bolibrukh's estimate [Bol95, Proposition 1.2.3, p. 24]

$$
\sum_{s \in \mathbb{P}^{1}(\mathbb{C})} \sum_{i=1}^{n} e_{i}^{s} \leqslant 0
$$

The results of this paper are a slight improvement on those which have been announced in [Cor01b]. A French translation of the initial version of this paper can be obtained as [Cor01c].

\section{Local connections}

Let $K$ be a local valued field, complete with respect to its discrete valuation $v$. Denote by $\mathcal{O}$ its valuation ring. An element $t \in K$ is called a uniformizing parameter if it satisfies $v(t)=1$. Let $\Omega$ be a free $\mathcal{O}$-module of rank one and $d: \mathcal{O} \longrightarrow \Omega$ be a derivation such that there exists a uniformizing parameter $t$ whose derivation $d t$ is an $\mathcal{O}$-basis of $\Omega$ (cf. [Del70]). We will usually call $\Omega$ the module of differential 1-forms. Define furthermore $\Omega^{*}$ to be the $\mathcal{O}$-dual of $\Omega$, and let their respective vector spaces be $\Omega_{K}=\Omega \otimes_{\mathcal{O}} K$, the $K$-vector space of differential 1-forms, and $\Omega_{K}^{*}=\Omega^{*} \otimes_{\mathcal{O}} K$. For any $\tau \in \Omega_{K}^{*}$, denote with $\partial_{\tau}$ the map

$$
\begin{aligned}
\partial_{\tau}: K & \longrightarrow K \\
f & \longmapsto\langle d f, \tau\rangle .
\end{aligned}
$$

Given a uniformizing parameter $t$, there exists for any $f \in K$ a unique $\alpha_{f} \in K$ such that

$$
d f=\alpha_{f} d t
$$

The mapping $f \longmapsto \alpha_{f}$ is a derivation of $K$. We will thus write $\alpha_{f}=d f / d t$ and $\partial_{d t}=d / d t$. Denote with $\mathcal{D}_{K}=K d / d t$ the $K$-vector space of such derivations of $K$. There is a natural valuation, also denoted by $v$, on all these spaces.

Let $V$ be a $K$-vector space of finite dimension $n$. A linear connection on $V$ is an additive map

$$
\nabla: V \longrightarrow V \otimes_{K} \Omega_{K}
$$

satisfying Leibniz's rule

$$
\nabla(f v)=v \otimes d f+f \nabla v \quad \text { for all } f \in K \text { and all } v \in V .
$$

For any derivation $\partial \in \mathcal{D}_{K}$, one defines a map $\nabla_{\partial}: V \longrightarrow V$ by composing $\nabla$ with

$$
\begin{aligned}
V \otimes_{K} \Omega_{K} & \longrightarrow V \\
v \otimes \omega & \longmapsto\langle\omega, \partial\rangle v=\omega(\partial) v .
\end{aligned}
$$

The additive map $\nabla_{\partial}$ is a differential operator on $V$ : it satisfies the relation

$$
\nabla_{\partial}(f v)=\partial(f) v+f \nabla_{\partial}(v) \quad \text { for all } f \in K \text { and all } v \in V \text {. }
$$




\section{ON FUCHS' RELATION FOR LINEAR DIFFERENTIAL SYSTEMS}

For a given choice of a uniformizing parameter $t$, we will mainly work with the derivations $d / d t$ and $\theta_{t}=t d / d t$. When no confusion can arise we will simply write $\theta$.

A vector $v \in V$ is said to be horizontal for the connection $\nabla$ if it satisfies $\nabla(v)=0$, which amounts to asking that $\nabla_{\partial}(v)=0$ for every derivation $\partial \in \mathcal{D}_{K}$.

For any basis $(e)$ of $V$, denote with $e_{i}$ the $i$ th vector of $(e)$. The matrix $\operatorname{Mat}\left(\nabla_{\partial},(e)\right)$ of the differential operator $\nabla_{\partial}$ in the basis $(e)$ is defined as the matrix $A=\left(A_{i j}\right) \in \mathrm{M}_{n}(K)$ such that

$$
\nabla_{\partial}\left(e_{j}\right)=-\sum_{i=1}^{n} A_{i j} e_{i} \text { for all } j=1, \ldots, n .
$$

Let $X={ }^{\mathrm{t}}\left(x_{1}, \ldots, x_{n}\right)$ be the vector of components of $v \in V$ in the basis $(e)$. The vector of components of $\nabla_{\partial}(v)$ in $(e)$ is then $\partial X-A X$. The differential system $\partial X=A X$ and the equation $\nabla_{\partial}(v)=0$ are therefore equivalent via the choice of a basis.

Let $(\varepsilon)$ be a basis of $V$ and let $P \in \mathrm{GL}_{n}(K)$ be the matrix of the basis change from $(e)$ to $(\varepsilon)$. The components of $v$ in $(\varepsilon)$ are then given by $Y={ }^{\mathrm{t}}\left(y_{1}, \ldots, y_{n}\right)$ where $X=P Y$, and the components of the vector $\nabla_{\partial}(v)$ by $\partial Y-A_{[P]} Y$, where the matrix $A_{[P]}$ is given by the so-called gauge transformation (with respect to the derivation $\partial$ )

$$
A_{[P]}=P^{-1} A P-P^{-1} \partial P .
$$

Until $\S 3$ we shall consider a fixed uniformizing parameter $t$ of $K$.

\subsection{Connections and constructions}

The constructions of a vector space endowed with a connection $(V, \nabla)$ are the spaces obtained by any finite succession of duality and quotient operations as well as tensor, exterior or symmetrical products. Any construction $C(V)$ of $(V, \nabla)$ is endowed with a natural connection $C(V)$ (cf. [Man65]). We will mainly be concerned with the following three constructions.

The connection $\nabla^{*}$ induced by $\nabla$ on the $K$-dual $V^{*}$ of $V$ is given for any $\partial \in \mathcal{D}_{K}$ by

$$
\nabla_{\partial}^{*}(f)(v)=\partial(f(v))-f\left(\nabla_{\partial}(v)\right) \quad \text { for any } f \in V^{*} \text { and any } v \in V .
$$

Let $(e)$ be a basis of $V$ and $A=\operatorname{Mat}\left(\nabla_{\partial},(e)\right)$ be the matrix of $\nabla_{\partial}$ in $(e)$. The matrix of $\nabla_{\partial}^{*}$ in the dual basis $\left(e^{*}\right)$ is then

$$
\operatorname{Mat}\left(\nabla_{\partial}^{*},\left(e^{*}\right)\right)=-{ }^{\mathrm{t}} A .
$$

The induced connection on End $V=V \otimes V^{*}$ is given by

$$
\text { End } \nabla_{\partial}(f)(v)=\nabla_{\partial}(f(v))-f\left(\nabla_{\partial}(v)\right) \quad \text { for any } f \in \text { End } V \text { and any } v \in V \text {. }
$$

The matrix of End $\nabla_{\partial}$ in the basis $\left(e \otimes e^{*}\right)$ then satisfies

$$
\operatorname{Mat}\left(\operatorname{End} \nabla_{\partial},\left(e \otimes e^{*}\right)\right)=A \otimes I-I \otimes{ }^{\mathrm{t}} A \text {. }
$$

The maximal exterior power $\bigwedge^{n} V$ is endowed with the connection defined by

$$
\bigwedge^{n} \nabla_{\partial}\left(v_{1} \wedge \cdots \wedge v_{n}\right)=\nabla_{\partial}\left(v_{1}\right) \wedge \cdots \wedge v_{n}+\cdots+v_{1} \wedge \cdots \wedge \nabla_{\partial}\left(v_{n}\right)
$$

for any $\left(v_{1}, \ldots, v_{n}\right) \in V^{n}$. The corresponding matrix is the scalar

$$
\operatorname{Mat}\left(\bigwedge^{n} \nabla_{\partial}, e_{1} \wedge \cdots \wedge e_{n}\right)=\operatorname{Tr} A
$$




\section{E. COREL}

\section{Lattices of vector spaces endowed with a connection}

For any free $\mathcal{O}$-module of finite type $M$ of $V$, define the rank of $M$ as the minimum number rk $M$ of generators for $M$.

Definition 1. Let $V$ be a $K$-vector space of dimension $n$. We say that $M$ is:

1) a lattice of $V$ if $M$ is a free $\mathcal{O}$-module of rank $n$ of $V$;

2) a sublattice of a lattice $\Lambda$ if $M$ is a lattice of $V$ included in $\Lambda$;

3) a partial lattice of $V$ if $M$ is a free $\mathcal{O}$-module of finite type (generally of rank $<n$ ) of $V$, and a partial sublattice of $\Lambda$ if it is a partial lattice included in $\Lambda$;

4) the free $\mathcal{O}$-module of rank $r$ spanned by $(e)$ if $M=\bigoplus_{i=1}^{r} \mathcal{O} e_{i}$. We write then $M=\mathcal{L}(e)$ and say that $(e)$ is a $(\mathcal{O}$-) basis of $M$.

We denote with $\mathcal{L}$ the set of lattices of $V$.

Lemma 2.1. Let $\Lambda$ be a lattice of $V$.

i) For any $r$-dimensional vector subspace $W$ of $V$, the $\mathcal{O}$-module $M=\Lambda \cap W$ is a lattice of $W$ and a partial sublattice of $\Lambda$.

ii) Let $\varphi$ be a $K$-automorphism of $V$. The image $\varphi(\Lambda)$ of $\Lambda$ is a lattice, and $\varphi(\Lambda) \subset \Lambda$ (respectively $\varphi(\Lambda)=\Lambda$ ) if and only if there exists a basis $(e)$ of $\Lambda$ such that $\operatorname{Mat}(\varphi,(e)) \in$ $\mathrm{M}_{n}(\mathcal{O})$ (respectively $\operatorname{Mat}(\varphi,(e)) \in \mathrm{GL}_{n}(\mathcal{O})$ ). This last condition then holds for any basis $(e)$ of $\Lambda$.

Definition 2. The connection $\nabla$ is said to be regular if there exists a lattice of $V$ which is stable under $\nabla_{\theta}$. The connection is said to be irregular otherwise.

\subsection{Valuation defined by a lattice}

Let $\Lambda$ be a lattice of $V$. We define a valuation $v_{\Lambda}$ on $V$ by letting

$$
v_{\Lambda}(x)=\sup \left\{k \in \mathbb{Z} \mid x \in t^{k} \Lambda\right\} \quad \text { for any } x \in V .
$$

For any lattice $M$ of $V$, and more generally for any non-empty subset $M$ of a lattice, we put

$$
v_{\Lambda}(M)=\inf _{x \in M} v_{\Lambda}(x),
$$

agreeing that $v_{\Lambda}(M)=\infty$ if $M=(0)$.

Lemma 2.2. Let $\Lambda$ be a lattice of $V$.

i) $v_{\Lambda}(x+\tilde{x}) \geqslant \min \left(v_{\Lambda}(x), v_{\Lambda}(\tilde{x})\right)$ holds for all $x, \tilde{x} \in V$.

ii) Let $W$ be a vector subspace of $V$, and $M \subset \Lambda \cap W$ a partial sublattice of $\Lambda$. Then the inequality $v_{M}(x) \leqslant v_{\Lambda}(x)$ holds for any $x \in W$.

iii) Let $M$ and $\tilde{M}$ be two partial sublattices of $\Lambda$. Then we have

$$
v_{\Lambda}(M+\tilde{M})=\min \left(v_{\Lambda}(M), v_{\Lambda}(\tilde{M})\right) .
$$

Proof. Consider $x$ and $\tilde{x}$ in $V$. One has

$$
\begin{aligned}
\min \left(v_{\Lambda}(x), v_{\Lambda}(\tilde{x})\right) & =\sup \left\{k \in \mathbb{Z} \mid x \in t^{k} \Lambda \text { and } \tilde{x} \in t^{k} \Lambda\right\} \\
& \leqslant \sup \left\{k \in \mathbb{Z} \mid x+\tilde{x} \in t^{k} \Lambda\right\}=v_{\Lambda}(x+\tilde{x}),
\end{aligned}
$$

hence part i follows. Let $x \in W$. If $x \in t^{k} M$, then $x \in t^{k} \Lambda$, and thus we get

$$
v_{M}(x)=\sup \left\{k \in \mathbb{Z} \mid x \in t^{k} M\right\} \leqslant \sup \left\{k \in \mathbb{Z} \mid x \in t^{k} \Lambda\right\}=v_{\Lambda}(x),
$$




\section{ON FUCHS' RELATION FOR LINEAR DIFFERENTIAL SYSTEMS}

and so part ii is proved. Let $M$ and $\tilde{M}$ be two partial sublattices of $\Lambda$. According to part i, we have

$$
v_{\Lambda}(M+\tilde{M})=\inf _{x \in M+\tilde{M}} v_{\Lambda}(x) \geqslant \min \left(v_{\Lambda}(M), v_{\Lambda}(\tilde{M})\right) .
$$

On the other hand, since $M \subset M+\tilde{M}$, we get $v_{\Lambda}(M) \leqslant v_{\Lambda}(M+\tilde{M})$. The same result holds with $\tilde{M}$, and hence part iii follows.

\subsection{Lattice invariants}

The theorem of elementary divisors holds in the principal domain $\mathcal{O}$. For any lattice $\Lambda$ of $V$, and any free $\mathcal{O}$-submodule $M$ of rank $r$ of $\Lambda$, there exists a unique increasing sequence of integers $k_{1} \leqslant \cdots \leqslant k_{r}$ and an $\mathcal{O}$-basis $\left(e_{1}, \ldots, e_{n}\right)$ of $\Lambda$ such that $\left(t^{k_{1}} e_{1}, \ldots, t^{k_{r}} e_{r}\right)$ is a basis of $M$.

In the general case, the partial lattice $t^{-v_{\Lambda}(M)} M$ is a submodule of $\Lambda$. A partial lattice of $V$ thus always has such a basis.

Definition 3. Let $\Lambda$ be a lattice of $V$. For any free $\mathcal{O}$-module $M$ of rank $r$ of $V$, we give the following definitions.

i) We call elementary divisors of $M$ in $\Lambda$ the integers

$$
k_{1}=\ell_{1}+v_{\Lambda}(M), \ldots, k_{r}=\ell_{r}+v_{\Lambda}(M)
$$

where $t^{\ell_{1}}, \ldots, t^{\ell_{r}}$ are the elementary divisors of $t^{-v_{\Lambda}(M)} M$ in $\Lambda$ in the usual sense.

ii) We call Smith basis of $\Lambda$ for $M$ any basis (e) of $\Lambda$ such that $\left(t^{k_{1}} e_{1}, \ldots, t^{k_{r}} e_{r}\right)$ form a basis of $M$.

We will write the elementary divisors of $M$ in $\Lambda$ as $k_{i, \Lambda}(M)$ to specify if necessary the respective $\mathcal{O}$-modules, and let

$$
\mathcal{E}_{\Lambda}(M)=\left(k_{1, \Lambda}(M), \ldots, k_{r, \Lambda}(M)\right)
$$

Proposition 2.1. Let $N \subset M$ be two lattices of $V$, and $\Lambda$ be any lattice of $V$. The respective elementary divisors of $M$ and $N$ in $\Lambda$ satisfy

$$
k_{i, \Lambda}(M) \leqslant k_{i, \Lambda}(N) \quad \text { for any } i=1, \ldots, n .
$$

Proof. Let $P$ be the matrix of the basis change from a Smith basis for $M$ to a Smith basis for $N$ in $\Lambda$. The matrix $t^{-\mathcal{E}_{\Lambda}(M)} P t^{\mathcal{E}_{\Lambda}(N)}$ is the matrix of the basis change from a basis of $M$ to a basis of $N$. Accordingly, Lemma 2.1, part ii yields

$$
v\left(P_{i j} t^{k_{j, \Lambda}(N)-k_{i, \Lambda}(M)}\right) \geqslant 0 \text { for any } 1 \leqslant i, j \leqslant n .
$$

Since $P \in \mathrm{GL}_{n}(\mathcal{O})$, there exists a permutation $\sigma$ such that $v\left(P_{i \sigma(i)}\right)=0$ for all $i=1, \ldots, n$. The relation $k_{\sigma(i), \Lambda}(N) \geqslant k_{i, \Lambda}(M)$ follows. The two sequences increase, hence we have

$$
k_{i, \Lambda}(N) \geqslant k_{i, \Lambda}(M) \text {. }
$$

The index of a sublattice $M$ in the lattice $\Lambda$ is defined as the (finite) length

$$
[\Lambda: M]=\chi(\Lambda / M)
$$

of the quotient module $\Lambda / M$ (cf. [Ser68, Part III, $\S 1$, p. 58]).

Lemma 2.3. Let $\Lambda \supset M$ be two lattices of $V$. Then the following hold:

i) $[\Lambda: M]=\sum_{i=1}^{n} k_{i, \Lambda}(M)=v(\operatorname{det} P)$ for any gauge matrix $P$ from $\Lambda$ to $M$.

ii) If $N$ is a sublattice of $M$, we have $[\Lambda: N]=[\Lambda: M]+[M: N]$. 


\section{E. Corel}

Corollary 2.1. Let $W$ and $\tilde{W}$ be two supplementary subspaces of $V$ of respective dimensions $m=\operatorname{dim}_{K} W$ and $p=\operatorname{dim}_{K} \tilde{W}$. Let $\Lambda \supset M$ be two lattices of $W$ and $\tilde{\Lambda} \supset \tilde{M}$ be two lattices of $\tilde{W}$. Then

$$
[\Lambda \oplus \tilde{\Lambda}: M \oplus \tilde{M}]=\sum_{i=1}^{m} k_{i, \Lambda}(M)+\sum_{i=1}^{p} k_{i, \tilde{\Lambda}}(\tilde{M})
$$

The Poincaré rank of a system $d X / d t=A X$ is the integer $-v(A)-1$. Since it is invariant under gauge transformations in $\mathrm{GL}_{n}(\mathcal{O})$, it is an invariant of the spanned lattice.

Definition 4. We call Poincaré rank of the connection $\nabla$ on the lattice $\Lambda$ the integer

$$
\mathfrak{p}_{\Lambda}(\nabla)=-v_{\Lambda}\left(\Lambda+\nabla_{\theta}(\Lambda)\right) .
$$

Definition 5. We call, after Gérard and Levelt [GL73], order of the singularity of $\nabla$ the minimum Poincaré rank

of the connection $\nabla$.

$$
m(\nabla)=\min _{M \in \mathcal{L}} \mathfrak{p}_{M}(\nabla)
$$

Remark 3. In the case where $\nabla$ is a regular connection, the order of the singularity is $m(\nabla)=0$.

Definition 6. Let $\Lambda$ be a lattice of $V$ and let $\mathfrak{p}=\mathfrak{p}_{\Lambda}(\nabla)$ be the Poincaré rank of $\nabla$ on $\Lambda$. We call polar map the map $\bar{\nabla}^{\Lambda}$ induced on $\Lambda / t \Lambda$ by the operator $t^{\mathfrak{p}} \nabla_{\theta}$. If $\Lambda$ is $\nabla_{\theta^{-}}$-stable, we call $\bar{\nabla}^{\Lambda}$ the residue $\operatorname{Res}_{\Lambda} \nabla$ of $\nabla$ on the lattice $\Lambda$.

Even when the residue is not defined, its trace is well defined. We denote by $\tau_{\Lambda}(\nabla)$ the corresponding invariant of the lattice $\Lambda$.

Definition 7. We call residue trace of the connection $\nabla$ on the lattice $\Lambda$ the complex number

$$
\tau_{\Lambda}(\nabla)=\bigwedge^{n} \Lambda^{n} \Lambda
$$

Lemma 2.4. Let $M \subset \Lambda$ be two lattices of $V$. The index of $M$ in $\Lambda$ satisfies

$$
[\Lambda: M]=\tau_{\Lambda}(\nabla)-\tau_{M}(\nabla) .
$$

Proof. Let $(e)$ be a basis of $\Lambda$ and $(\varepsilon)$ a basis of $M$. Let $P \in \mathrm{GL}_{n}(K)$ be the gauge matrix from $(e)$ to $(\varepsilon)$. Let $A=\operatorname{Mat}\left(\nabla_{d / d t},(e)\right)$ and $B=\operatorname{Mat}\left(\nabla_{d / d t}(\varepsilon)\right)$. The gauge equation $d / d t P=A P-P B$ implies that

Taking residues at $t=0$ yields

$$
\frac{d}{d t}(\operatorname{det} P)=(\operatorname{Tr} A-\operatorname{Tr} B) \operatorname{det} P
$$

$$
v(\operatorname{det} P)=\operatorname{Tr} \operatorname{Res}_{t=0} A-\operatorname{Tr} \operatorname{Res}_{t=0} B .
$$

\subsection{Subspaces and lattices}

Let $\left(V_{i}\right)_{1 \leqslant i \leqslant s}$ be a family of $K$-vector subspaces of $V$ of respective dimensions $n_{i}=\operatorname{dim}_{K} V_{i}$ such that

$$
V=\bigoplus_{i=1}^{s} V_{i}
$$




\section{ON FUCHS' RELATION FOR LINEAR DIFFERENTIAL SYSTEMS}

The direct sum $\bigoplus_{i=1}^{s} M \cap V_{i}$ is a sublattice of $M$, but, according to the position of $M$ with respect to the $V_{i}$, one is not sure to recover the lattice $M$ itself.

Definition 8. A lattice $M$ of $V$ is said to be compatible with the direct sum $\bigoplus_{i=1}^{s} V_{i}$ if

$$
M=\bigoplus_{i=1}^{s}\left(M \cap V_{i}\right) .
$$

Proposition 2.2. Let $M$ be a lattice of $V$. The lattice $\bigoplus_{i=1}^{s}\left(M \cap V_{i}\right)$ is the largest sublattice of $M$ compatible with the direct sum $\bigoplus_{i=1}^{s} V_{i}$.

Proof. The lattice $\bigoplus_{i=1}^{s}\left(M \cap V_{i}\right)$ is compatible with the direct sum $\bigoplus_{i=1}^{s} V_{i}$ according to its construction. Let $N$ be a lattice of $V$ compatible with the direct sum $\bigoplus_{i=1}^{s} V_{i}$ and satisfying $\bigoplus_{i=1}^{s}\left(M \cap V_{i}\right) \subset N \subset M$. Their restrictions to $V_{i}$ satisfy $M \cap V_{i} \subset N \cap V_{i} \subset M \cap V_{i}$ for all $i=1, \ldots, n$. Thus $M \cap V_{i}=N \cap V_{i}$ and so the equality $\bigoplus_{i=1}^{s}\left(M \cap V_{i}\right)=\bigoplus_{i=1}^{s}\left(N \cap V_{i}\right)=N$ follows.

Lemma 2.5. Let $M$ and $\tilde{M}$ be two lattices of $V$. The Poincaré rank of the connection $\nabla$ on $M+\tilde{M}$ satisfies

$$
\mathfrak{p}_{M+\tilde{M}}(\nabla) \leqslant \max \left(\mathfrak{p}_{M}(\nabla), \mathfrak{p}_{\tilde{M}}(\nabla)\right) .
$$

In particular, if $M$ and $\tilde{M}$ are $\nabla_{\theta}$-stable, then the same holds for $M+\tilde{M}$.

Proof. By definition $\mathfrak{p}_{M+\tilde{M}}(\nabla)=-v_{M+\tilde{M}}\left(M+\tilde{M}+\nabla_{\theta}(M+\tilde{M})\right)$. According to Lemma 2.2, part iii, one has

$$
v_{M+\tilde{M}}\left(M+\tilde{M}+\nabla_{\theta}(M+\tilde{M})\right)=\min \left(v_{M+\tilde{M}}\left(M+\nabla_{\theta}(M)\right), v_{M+\tilde{M}}\left(\tilde{M}+\nabla_{\theta}(\tilde{M})\right)\right) .
$$

Since $M \subset M+\tilde{M}$, we get $v_{M+\tilde{M}}\left(M+\nabla_{\theta}(M)\right) \geqslant v_{M}\left(M+\nabla_{\theta}(M)\right)$. Similarly, one has

$$
v_{M+\tilde{M}}\left(\tilde{M}+\nabla_{\theta}(\tilde{M})\right) \geqslant v_{\tilde{M}}\left(\tilde{M}+\nabla_{\theta}(\tilde{M})\right) .
$$

Corollary 2.2. Let $m=m(\nabla)$ be the order of the singularity of the connection $\nabla$. For any $k \geqslant m$, and any lattice $\Lambda$ of $V$, there exists a unique maximal sublattice $\Lambda_{k}$ of $\Lambda$ such that $\mathfrak{p}_{\Lambda_{k}}(\nabla) \leqslant k$.

Proof. Let $M$ be a lattice of $V$ such that $\mathfrak{p}_{M}(\nabla)=m$. The Poincaré rank of $\nabla$ on the lattice $t^{-v_{\Lambda}(M)} M$ is equal to $\mathfrak{p}_{t^{-v_{\Lambda}(M)} M}(\nabla)=m \leqslant k$, thus the set $\mathcal{L}_{k}$ of all sublattices of $\Lambda$ of Poincaré rank $\leqslant k$ is non-empty. Since $\Lambda$ is a module of finite type on the principal domain $\mathcal{O}$, the sum of all elements of $\mathcal{L}_{k}$ is still a sublattice of $\Lambda$, and according to Lemma 2.5, the Poincaré rank on this lattice is also $\leqslant k$. Hence

$$
\Lambda_{k}=\sum_{\substack{M \subset \Lambda \\ \mathfrak{p}_{M}(\nabla) \leqslant k}} M
$$

is the largest sublattice of $\Lambda$ of Poincaré rank $\leqslant k$.

Remark 4 . In the case where $\nabla$ is a regular connection, the lattice $\Lambda_{0}$ exists and is equal to the Levelt lattice $\Lambda_{L}$ of $\Lambda$ that we defined in [Cor99b].

Recall the construction of Gérard and Levelt [GL73] of a saturated lattice. Let $\Lambda$ be a lattice of $V$ and $\vartheta \in \mathcal{D}_{K}$ be a derivation of $K$. One calls the $k$ th saturated lattice of $\Lambda$ with respect to $\vartheta$ the lattice

$$
\mathcal{F}_{\vartheta}^{k}(\Lambda)=\Lambda+\nabla_{\vartheta}(\Lambda)+\cdots+\nabla_{\vartheta}^{k}(\Lambda) \text { for any } k \in \mathbb{N} .
$$

It is possible to determine the order of the singularity of $\nabla$ with these lattices by means of the following result. 


\section{E. COREL}

Theorem 2 (Gérard-Levelt). If the connection $\nabla$ has order of singularity $m$, then for every lattice $\Lambda$ of $V$, the $(n-1)$ th saturated lattice $\mathcal{F}_{t^{k} \theta}^{n-1}(\Lambda)$ of $\Lambda$ is $t^{k} \nabla_{\theta}$-stable, for any $k \geqslant m$.

Remark 5. After a remark of Marius van der Put, one sees that the $(n-1)$ th saturated lattice $\mathcal{F}_{t^{k} \theta}^{n-1}(\Lambda)$ of $\Lambda$ is the smallest lattice of $V$ containing $\Lambda$ which is $t^{k} \nabla_{\theta^{-}}$stable.

\section{Canonical decompositions of connections}

Let us now consider complex analytic differential systems, and take $z$ as the standard coordinate of $\mathbb{C}$. The classical local theory of irregular singularities (e.g. [Huk37], [Tur55], [Rob80], [Jur78]) asserts that there exists a fundamental matrix of formal solutions for the system $z d Y / d z=A(z) Y$ satisfying $\mathcal{Y}=U(\zeta) \zeta^{p L} e^{Q(1 / \zeta)}$ where $\zeta^{p}=z$ for some $p \in \mathbb{N}, U$ is a square matrix of order $n$ with coefficients in $\mathbb{C}((X)), L$ is a constant matrix, and $Q$ is a diagonal matrix of polynomials in $X \mathbb{C}[X]$.

Let us now denote with $K=\mathbb{C}((z))$ the field of all formal meromorphic power series, with $\mathcal{O}=\mathbb{C}[[z]]$ the valuation ring of $K$ for its $z$-adic valuation $v$. One easily checks that the ordinary differentiation

$$
d: \mathcal{O} \longrightarrow \Omega_{\mathcal{O} \mid \mathbb{C}}^{1}
$$

where $\Omega_{\mathcal{O} \mid \mathbb{C}}^{1}$ is the $\mathcal{O}$-module of formal holomorphic differential 1-forms over $\mathbb{C}$, satisfies the assumptions of $\S 1$ with $z$ as uniformizing parameter. Denote further with $\Omega_{K \mid \mathbb{C}}^{1}$ the $K$-vector space of differential 1-forms over $\mathbb{C}$ and with $\operatorname{Der}_{\mathbb{C}}(K)$ the $K$-vector space of $\mathbb{C}$-derivations of $K$. The space $\operatorname{Der}_{\mathbb{C}}(K)$ is then the $K$-dual of $\Omega_{K \mid \mathbb{C}}^{1}$.

We consider all the definitions of $\S 2$ in this setting.

\subsection{Ramification}

The occurrence of rational powers of the variable $z$ in the formal solutions at an irregular singularity is already mentioned in Fabry's thesis in 1885 [Fab85]. It corresponds to finite algebraic extensions of the field $K$, accounting for the ramification of the system. We call ramification order of the system $z d Y / d z=A(z) Y$ the smallest integer $p$ such that there exists a formal solution under the above mentioned form. According to Levelt [Lev75], there is an a priori upper bound for $p$.

Proposition 3.1 (Levelt). The ramification order of a system of order $n$ is smaller than $\operatorname{lcm}(1,2, \ldots, n)$.

Let $p \in \mathbb{N}$. We denote with $H$ the extension $K[T] /\left(T^{p}-z\right)$ of $K$. There exists a unique extension of the differential $d$ of $K$ to $H$, that we also denote with $d$

$$
d: H \longrightarrow \Omega_{H \mid \mathbb{C}}^{1}=\Omega_{K \mid \mathbb{C}}^{1} \otimes_{K} H .
$$

We extend in a unique way the connection $\nabla$ to the space $V_{H}=V \otimes_{K} H$ by letting

$$
\nabla_{H}=\nabla \otimes 1+\mathrm{id}_{V} \otimes d .
$$

We identify $V$ to the $K$-subspace $V \otimes 1$ of $V_{H}$.

By calling $\zeta$ the class of $T$ in the field $H$ we get a natural isomorphism $H \simeq \mathbb{C}((\zeta))$. The valuation $v$ of $K$ extends in a unique way to a discrete valuation of $H$, that we also denote by $v: H \longrightarrow(1 / p) \mathbb{Z}$, which satisfies $v(\zeta)=1 / p$. This valuation does not coincide with the $\zeta$-adic valuation $w$ on $H$ which takes its values in $\mathbb{Z}$. The valuation $\operatorname{ring} \mathcal{O}_{H}$ of $H$ for these two valuations is the same, because $w=p v$. For any lattice $M$ of $V_{H}$, we denote with $v_{M}$ the valuation induced by $v$ and with $w_{M}$ the valuation induced by $w$ on $V_{H}$, which satisfies $w_{M}=p v_{M}$. To every lattice $\Lambda$ of $V$ there corresponds 


\section{ON FUCHS' RELATION FOR LINEAR DIFFERENTIAL SYSTEMS}

a lattice $\Lambda_{H}=\Lambda \otimes_{\mathcal{O}} \mathcal{O}_{H}$ of $V_{H}$. We shall identify $\Lambda$ to the $\mathcal{O}$-submodule $\Lambda \otimes 1$ of $\Lambda_{H}$. Through this identification, the valuation $v_{\Lambda_{H}}$ of $V_{H}$, restricted to $V \otimes 1$, coincides with $v_{\Lambda}$.

Since $\zeta$ is a uniformizing parameter of $H$, every notion defined in $\S 2$ makes sense for the lattices of $\left(V_{H}, \nabla_{H}\right)$. However, since the differential 1-form $d z / z$ satisfies

$$
\frac{d z}{z}=p \frac{d \zeta}{\zeta}
$$

and can be defined as an element of $\Omega_{H \mid \mathbb{C}}^{1}$, the operator $\nabla_{\theta}$ thus also extends to an operator $\left(\nabla_{\theta}\right)_{H}$ of $V_{H}$. One checks easily that $\left(\nabla_{\theta}\right)_{H}=\left(\nabla_{H}\right)_{\theta}$ holds. We will frequently drop the index and write simply $\nabla$. The two derivations $\theta=z d / d z$ and $\theta_{\zeta}=\zeta d / d \zeta$ of $H$ satisfy $\theta_{\zeta}=p \theta$. Therefore, we have

$$
\nabla_{\theta_{\zeta}}=p \nabla_{\theta}
$$

Considering the two-foldedness of these definitions, we will write with a $\zeta$ index every object defined in $\S 2$ with respect to $\zeta$ as a uniformizing parameter.

Lemma 3.1. Let $\Lambda$ be a lattice of $V$ and $M$ be a lattice of $V_{H}$.

i) $M$ is $\nabla_{\theta}$-stable if and only if $M$ is $\nabla_{\theta_{\zeta}}$-stable.

ii) $\Lambda$ is $\nabla_{\theta}$-stable if and only if $\Lambda_{H}$ is $\nabla_{\theta}$-stable.

iii) If $\Lambda$ is $\nabla_{\theta}$-stable, the residue $\left(\operatorname{Res}_{\zeta}\right)_{\Lambda_{H}} \nabla$ induced by $\nabla_{\theta_{\zeta}}$ on $\Lambda_{H} / \zeta \Lambda_{H}$ satisfies

$$
\operatorname{Mat}\left(\left(\operatorname{Res}_{\zeta}\right)_{\Lambda_{H}} \nabla,(\overline{e \otimes 1})\right)=p \operatorname{Mat}\left(\operatorname{Res}_{\Lambda} \nabla,(\bar{e})\right) \quad \text { for any basis }(e) \text { of } \Lambda,
$$

where $(\bar{e})$ denotes the quotient basis of $\Lambda$ and $(\overline{e \otimes 1})$ denotes the corresponding quotient basis of $\Lambda_{H} / \zeta \Lambda_{H}$.

iv) The Poincaré rank $\left(\mathfrak{p}_{\zeta}\right)_{\Lambda_{H}}(\nabla)=-w_{\Lambda_{H}}\left(\Lambda_{H}+\nabla_{\theta_{\zeta}}\left(\Lambda_{H}\right)\right)$ of $\nabla$ on the lattice $\Lambda_{H}$ satisfies

$$
\left(\mathfrak{p}_{\zeta}\right)_{\Lambda_{H}}(\nabla)=p \mathfrak{p}_{\Lambda}(\nabla) \text {. }
$$

Proof. Let $\left(\varepsilon_{1}, \ldots, \varepsilon_{n}\right)$ be a basis on $\mathcal{O}_{H}$ of $M$. Since $\theta_{\zeta}=p \theta$, the respective matrices of $\nabla_{\theta}$ and $\nabla_{\theta_{\zeta}}$ in $(\varepsilon)$ satisfy

$$
\operatorname{Mat}\left(\nabla_{\theta_{\zeta}},(\varepsilon)\right)=p \operatorname{Mat}\left(\nabla_{\theta},(\varepsilon)\right),
$$

whence we get part i. Let $(e)=\left(e_{1}, \ldots, e_{n}\right)$ be an $\mathcal{O}$-basis of $\Lambda$. From the equality

$$
\Lambda_{H}=\bigoplus_{i=1}^{n} \mathcal{O}_{H} e_{i} \otimes 1
$$

we get $\nabla_{\theta}\left(e_{i} \otimes 1\right)=\nabla_{\theta}\left(e_{i}\right) \otimes 1+e_{i} \otimes \theta(1)=\nabla_{\theta}\left(e_{i}\right) \otimes 1$ and part ii follows. One also has

$$
\nabla_{\theta_{\zeta}}\left(e_{i} \otimes 1\right)=\left\langle\nabla\left(e_{i}\right) \otimes 1+e_{i} \otimes d(1), \theta_{\zeta}\right\rangle=p \nabla_{\theta}\left(e_{i}\right) \otimes 1 .
$$

The matrix of the connection $\nabla_{\theta_{\zeta}}$ in $\left(e_{i} \otimes 1\right)$ thus satisfies

$$
\operatorname{Mat}\left(\nabla_{\theta_{\zeta}},(e \otimes 1)\right)=p \operatorname{Mat}\left(\nabla_{\theta},(e)\right)
$$

which proves part iii. Write $A=\operatorname{Mat}\left(\nabla_{\theta},(e)\right)$. The Poincaré rank $\left(\mathfrak{p}_{\zeta}\right)_{\Lambda_{H}}(\nabla)$ satisfies

$$
\left(\mathfrak{p}_{\zeta}\right)_{\Lambda_{H}}(\nabla)=\min _{i, j} w\left(p A_{i j}\right)=\min _{i, j} p v\left(p A_{i j}\right)=p \mathfrak{p}_{\Lambda}(\nabla),
$$

which concludes the proof.

We wish to extend the invariants defined on $K$ to $V_{H}$. The former proof shows that we must choose the valuation $v$ and the derivation $\theta$. However, the residue $\operatorname{Res}_{\zeta}$ for the connection $\nabla_{H}$ defined with respect to the uniformizing parameter $\zeta$ is not consistent with this choice. In order to obtain a definition compatible with the extensions, we set the following definitions. 


\section{E. Corel}

Definition 9. Let $M$ be a $\nabla_{\theta}$-stable lattice of $V_{H}$. We call compatible residue of $\nabla$ on $M$ the map $\operatorname{Res}_{M}^{c} \nabla$ of $M / \zeta M$ induced by the operator $\nabla_{\theta}$.

If $\Lambda$ is $\nabla_{\theta}$-stable, the compatible residue of $\nabla$ on $\Lambda_{H}$ satisfies

$$
\operatorname{Mat}\left(\operatorname{Res}_{\Lambda_{H}}^{c} \nabla,(\overline{e \otimes 1})\right)=\operatorname{Mat}\left(\operatorname{Res}_{\Lambda} \nabla,(\bar{e})\right),
$$

for any basis $(e)$ of $\Lambda$, with the notations of Lemma 3.1.

\subsection{The associated regular connection and the determinant map}

We show that a connection has the following canonical decomposition.

Theorem 3. Let $(V, \nabla)$ be a $K$-vector space endowed with a connection. There exists a unique regular connection $\nabla^{r}: V \longrightarrow V \otimes_{K} \Omega_{K \mid \mathbb{C}}^{1}$ such that the following holds.

i) The map $\varphi=\nabla_{\theta}-\nabla_{\theta}^{r}$ of $V$ is semi-simple, and its eigenvalues $\varphi_{i}$ belong to $\left(1 / z^{1 / p}\right) \mathbb{C}\left[1 / z^{1 / p}\right]$ for some $p \in \mathbb{N}$.

ii) The map $\left(\text { End } \nabla^{r}\right)_{\theta}(\varphi)=\left[\nabla_{\theta}, \varphi\right]$ of $V$ commutes with $\varphi$.

The smallest such $p \in \mathbb{N}$ is called the ramification order of the connection $\nabla$. We denote with $\omega$ the $K$-linear map $\omega=\nabla-\nabla^{r}: V \longrightarrow V \otimes_{K} \Omega_{K \mid \mathbb{C}}^{1}$. We call $\nabla=\nabla^{r}+\omega$ the canonical decomposition of the connection $\nabla$.

Remark 6. This decomposition differs from the Jordan form given by Levelt in 1975 [Lev75, Theorem I, p. 9], who writes the operator $\nabla_{\theta}$ as a unique sum of a commuting semi-simple differential operator and nilpotent $K$-linear map.

The proof of Theorem 3 will be the subject of the following subsection $(\S 3.3)$.

DEFINITION 10. We respectively call regular connection associated to $\nabla$ and determinant endomorphism, the connection $\nabla^{r}$ and the map $\varphi=\omega_{\theta}$ described in Theorem 3. We call $\omega$ the determinant map of $\nabla$.

Lemma 3.2. Let $\nabla$ be a connection on $V$ and $\nabla=\nabla^{r}+\omega$ be the canonical decomposition of the connection $\nabla$. Then:

i) $\nabla^{*}=\left(\nabla^{r}\right)^{*}-\omega^{*}$;

ii) End $\nabla=$ End $\nabla^{r}+\left(\omega \otimes \mathrm{id}_{V^{*}}-\mathrm{id}_{V} \otimes \omega^{*}\right)$;

iii) $\bigwedge^{n} \nabla=\bigwedge^{n} \nabla^{r}+\operatorname{Tr} \omega$

are the canonical decompositions of the corresponding connections.

In the space $V_{H}$, endowed with the connection $\nabla_{H}$, we denote with $V_{i}$ the eigenspaces of $\varphi$ and $n_{i}=\operatorname{dim}_{H} V_{i}$ their respective dimensions for all $i=1, \ldots, s$. We denote with $\varphi_{i} \in\left(1 / z^{1 / p}\right) \mathbb{C}\left[1 / z^{1 / p}\right]$ the corresponding eigenvalues. We will call them attached eigenvalues of $\nabla$, and call determinant factors the primitives without constant term $Q_{i}=\int \varphi_{i} d z / z$ of these eigenvalues.

DeFinition 11. With the previous notations, we call Katz rank of the connection $\nabla$ the rational number

where $\bar{v}(x)=\min (v(x), 0)$.

$$
\kappa(\nabla)=-\min _{i=1, \ldots, s} \bar{v}\left(\varphi_{i}\right) \in \frac{1}{p} \mathbb{Z}
$$

Definition 12. We say that the vector space endowed with a connection $(V, \nabla)$ has only one determinant factor if its determinant endomorphism has only one eigenvalue. 


\section{ON FUCHS' RELATION FOR LINEAR DIFFERENTIAL SYSTEMS}

Corollary 3.1. The vector space endowed with a connection $\left(V_{H}, \nabla_{H}\right)$ is a canonical direct sum of subconnections having only one determinant factor. We call it the direct sum attached to the connection $\nabla$.

Proof. Denote with $\nabla_{i}=\left(\nabla_{H}\right)_{V_{i}}$ and $\nabla_{i}^{r}$ the restrictions of $\nabla_{H}$ and $\left(\nabla^{r}\right)_{H}$ to $V_{i}$. Condition ii of Theorem 3 implies that, for any derivation $\partial$ of $K$, the subspace $V_{i}$ remains stable under $\left(\nabla_{\partial}\right)_{H}$ for all $i=1, \ldots, s$. It is clear that

$$
\nabla_{i}=\nabla_{i}^{r}+\omega_{\left.\right|_{V_{i}}}
$$

is the canonical decomposition of $\nabla_{i}$. Since $\varphi_{\left.\right|_{V_{i}}}$ is scalar, $\left(V_{i}, \nabla_{i}\right)_{1 \leqslant i \leqslant s}$ is the claimed family of subconnections of $\left(V_{H}, \nabla_{H}\right)$.

\subsection{Canonical forms of Babbitt-Varadarajan: proof of Theorem 3}

Let us consider the derivation $\theta=z d / d z$. Let a formally meromorphic differential system

$$
\theta X=A X
$$

be given.

The following proposition by Babbitt-Varadarajan [BV83, Var91] explains which is the best reduced form (in the sense of Turrittin, see [Tur55]) of this system.

Proposition 3.2. For any matrix $A \in \mathrm{M}_{n}(K)$, there exists an integer $p \in \mathbb{N}$ and a gauge transformation $P \in \mathrm{GL}_{n}\left(\mathbb{C}\left(\left(z^{1 / p}\right)\right)\right)$ such that

$$
P^{-1} A P-P^{-1} \theta P=D_{r_{1}} z^{r_{1}}+\cdots+D_{r_{s}} z^{r_{s}}+C
$$

where:

i) $r_{1}<\cdots<r_{s}<0$ are distinct rational numbers such that $p r_{i} \in \mathbb{Z}$;

ii) any two matrices among $D_{r_{1}}, \ldots, D_{r_{s}}, C \in \mathrm{M}_{n}(\mathbb{C})$ commute;

iii) $D_{r_{1}}, \ldots, D_{r_{s}}$ are semi-simple;

iv) the eigenvalues of $C$ belong to the set $\{z \in \mathbb{C} \mid \operatorname{Re}(z) \in[0,1 / p[\}$.

The matrix $A$ is equivalent under gauge transformation in $\mathrm{GL}_{n}\left(\mathbb{C}\left(\left(z^{1 / p}\right)\right)\right)$ to a matrix

$$
D^{\prime}{ }_{r_{1}} z^{r_{1}}+\cdots+D^{\prime}{ }_{r_{s}} z^{r_{s}}+C^{\prime}
$$

satisfying conditions $i$ to iv, if and only if there exists $T \in \mathrm{GL}_{n}(\mathbb{C})$ such that:

a) $T^{-1} C T=C^{\prime}$;

b) $T^{-1} D_{r_{j}} T=D^{\prime}{ }_{r_{j}}$ for $1 \leqslant j \leqslant s$.

Such a matrix is called a $p$-reduced canonical form of the connection, and $D_{r_{1}} z^{r_{1}}+\cdots+D_{r_{s}} z^{r_{s}}$ is called the irregular part of the canonical form. The rational number $r_{1}$ is then equal to the Katz rank $\kappa(\nabla)$ of the connection $\nabla$.

Owing to the commutation condition ii, the system $\theta Z=A_{[P]}(z) Z$ has the matrix $\mathcal{Z}=$ $z^{C} \exp \left(\int D_{r_{1}} z^{r_{1}}+\cdots+D_{r_{s}} z^{r_{s}} d z / z\right)$ as a fundamental matrix of formal solutions. We can also write it under the following form

$$
\begin{aligned}
\mathcal{Y}=P \mathcal{Z} & =P\left(z^{1 / p}\right) z^{C} \exp \left(\frac{1}{r_{1}-1} D_{r_{1}} z^{r_{1}}+\cdots+\frac{1}{r_{s}-1} D_{r_{s}} z^{r_{s}}\right) \\
& =P(\zeta) \zeta^{p C} e^{Q(1 / \zeta)} .
\end{aligned}
$$

According to Proposition 3.1, we only need to ramify up to the order $\operatorname{lcm}(1,2, \ldots, n)$. Let us restate Proposition 3.2 as follows. 


\section{E. COREL}

Proposition 3.3. Let $\nabla$ be a connection on $V$. Let $p=\operatorname{lcm}(1,2, \ldots, n)$ and $H=K\left(z^{1 / p}\right)$. Let $\nabla_{H}$ be the unique extension of $\nabla$ to the space $V_{H}=V \otimes_{K} H$. Choose a pth root $\zeta$ of $z$. Let $\theta_{\zeta}$ be the derivation $\zeta d / d \zeta$. Then there exists a regular connection $\nabla^{r}$ on $V_{H}$, an $H$-linear map

$$
\omega: V_{H} \longrightarrow V_{H} \otimes_{H}\left(\Omega_{K \mid \mathbb{C}}^{1} \otimes_{K} H\right)
$$

and a basis $(\varepsilon)$ of $V_{H}$ such that the following four properties hold.

i) The matrix $\operatorname{Mat}\left(\nabla_{\theta_{\zeta}}^{r},(\varepsilon)\right)$ is a constant matrix $\tilde{C} \in \mathrm{M}_{n}(\mathbb{C})$ whose eigenvalues belong to the set $\{z \in \mathbb{C} \mid \operatorname{Re}(z) \in[0,1[\}$.

ii) The eigenvalues $\varphi_{i}$ of the map $\varphi=\left\langle\omega, \theta_{\zeta}\right\rangle$ are elements of $(1 / \zeta) \mathbb{C}[1 / \zeta]$ and $\varphi$ is diagonal in the basis $(\varepsilon)$.

iii) The map $\gamma=\nabla_{\theta_{\zeta}}^{r} \circ \varphi-\varphi \circ \nabla_{\theta_{\zeta}}^{r}$ satisfies $[\varphi, \gamma]=0$.

iv) $\nabla_{H}=\nabla^{r}+\omega$.

Proof. Let us show first of all that the result of Babbitt and Varadarajan implies Proposition 3.3. Let $(e)$ be a basis of $V$, and $A=\operatorname{Mat}(\nabla,(e))$. Let

$$
A_{[P]}=D_{r_{1}} z^{r_{1}}+\cdots+D_{r_{s}} z^{r_{s}}+C
$$

be a canonical form. Let us denote with $(\varepsilon)$ the basis to which the gauge transformation $P \in \mathrm{GL}_{n}(H)$ sends the basis $(e \otimes 1)$ of $V_{H}$. Define $\nabla^{r}$ as the connection whose matrix in $(\varepsilon)$ is $C \otimes d z / z$ and $\omega$ as the $H$-linear map whose matrix is $\left(D_{r_{1}} z^{r_{1}}+\cdots+D_{r_{s}} z^{r_{s}}\right) \otimes d z / z$ in $(\varepsilon)$. Since $d z / z=p d \zeta / \zeta$, one finds that $\operatorname{Mat}\left(\nabla_{\theta_{\zeta}}^{r},(\varepsilon)\right)=p C$, whose eigenvalues do belong to $\left\{z \in \mathbb{C} \mid \operatorname{Re}(z) \in\left[0,1[\}\right.\right.$. The $\varphi_{i}$ are the diagonal entries of $\tilde{D}=p D_{r_{1}} z^{r_{1}}+\cdots+p D_{r_{s}} z^{r_{s}}$. Therefore, we get

$$
\operatorname{Mat}(\gamma,(\varepsilon))=\theta_{\zeta} \tilde{D}+[p C, \tilde{D}] .
$$

Thus the matrix of the map $[\gamma, \varphi]$ satisfies

$$
\operatorname{Mat}([\gamma, \varphi],(\varepsilon))=\left[\theta_{\zeta} \tilde{D}, \tilde{D}\right]+[[p C, \tilde{D}], \tilde{D}]=[[p C, \tilde{D}], \tilde{D}] .
$$

Since the matrices $C$ and $D_{-j}$ commute for any $j=1, \ldots, m$, the statement $[\varphi, \gamma]=0$ holds by means of Lemma 3.3 stated below.

Conversely, let $\left(\nabla^{r}, \omega, \varepsilon\right)$ be a triple satisfying conditions i to iv. Denote with

$$
C=\operatorname{Mat}\left(\nabla_{\theta_{\zeta}}^{r},(\varepsilon)\right)
$$

the matrix of the operator $\nabla_{\theta_{\zeta}}^{r}$ and with

$$
D=\operatorname{Mat}\left(\left\langle\omega, \theta_{\zeta}\right\rangle,(\varepsilon)\right)=\operatorname{diag}\left(\varphi_{1}, \ldots, \varphi_{n}\right)=D_{-m} \zeta^{-m}+\cdots+D_{-1} \zeta^{-1},
$$

where the $D_{i}$ are constant diagonal matrices, the matrix of $\varphi=\left\langle\omega, \theta_{\zeta}\right\rangle$ in the basis $(\varepsilon)$. By assumption, the equalities

$$
\operatorname{Mat}\left(\nabla_{\theta_{\zeta}},(\varepsilon)\right)=D+C
$$

and

$$
\operatorname{Mat}([\gamma, \varphi],(\varepsilon))=[[C, D], D]=0
$$

hold. We want to show that $(1 / p)(D+C)$ is a canonical form of Babbitt-Varadarajan of $\nabla_{\theta}$. The second assumption yields

$$
[[C, D], D]=\left(C_{i j}\left(\varphi_{j}-\varphi_{i}\right)^{2}\right)=0 .
$$

Thus, whenever $\varphi_{j} \neq \varphi_{i}$, one has $C_{i j}=0$. Hence the matrices $C$ and $D$ commute. But, because $C$ is a constant matrix, this implies that $\left[C, D_{i}\right]=0$ for all $i$, and thus $(1 / p)(D+C)$ is a canonical form of Babbitt-Varadarajan of $\nabla_{\theta}$. 


\section{ON FUCHS' RELATION FOR LINEAR DIFFERENTIAL SYSTEMS}

We will now show that the decomposition stated in Proposition 3.3 is unique, then we will prove that this decomposition is in fact defined over the field $K$.

Let us work with a uniformizing parameter $t$ and denote with

$$
D_{-m} t^{-m}+\cdots+D_{-1} t^{-1}+C
$$

a canonical form. Let us start with three technical lemmas.

For a diagonal matrix $D=\left(D_{i}\right)$, denote with $I(D)$ the set of indexes

$$
I(D)=\left\{(i, j) \in\{1, \ldots, n\}^{2} \mid D_{i}=D_{j}\right\} .
$$

For an indexed matrix $P_{k}$, we will denote its elements with $\left(P_{i j}^{(k)}\right)$. If $D_{k}$ is diagonal, we will denote its elements with $\left(D_{i}^{(k)}\right)$.

Let $D_{0}, \ldots, D_{p}$ be diagonal matrices of $\mathrm{M}_{n}(\mathbb{C})$.

Lemma 3.3. A matrix $P \in \mathrm{M}_{n}(\mathbb{C})$ commutes with the matrices $D_{k}$ for all $k=1, \ldots, p$ if and only if

$$
P_{i j} \neq 0 \Longrightarrow(i, j) \in \bigcap_{k=0}^{p} I\left(D_{k}\right) .
$$

Proof. Indeed, for any $k$, the entries of the commutator matrices satisfy $\left[P, D_{k}\right]_{i j}=P_{i j}\left(D_{i}^{(k)}-D_{j}^{(k)}\right)$ for all $1 \leqslant i, j \leqslant n$.

We will denote with $S\left(D_{0}, \ldots, D_{p}\right)$ the system

$$
\begin{aligned}
{\left[D_{0}, X_{0}\right] } & =0, \\
{\left[D_{0}, X_{1}\right]+\left[D_{1}, X_{0}\right] } & =0, \\
& \vdots \\
{\left[D_{0}, X_{p}\right]+\cdots+\left[D_{p}, X_{0}\right] } & =0
\end{aligned}
$$

in the unknown matrices $\left(X_{0}, \ldots, X_{p}\right)$.

Lemma 3.4. If $\left(X_{0}, \ldots, X_{p}\right)$ satisfies the system $S\left(D_{0}, \ldots, D_{p}\right)$, then $X_{i}$ commutes with $D_{0}, \ldots, D_{p-i}$ for all $i=0, \ldots, p$.

Proof. The statement is obvious for $p=0$, so let us proceed by induction on the integer $p$. Assume that the statement is established for $p-1$. Let $\left(X_{0}, \ldots, X_{p}\right)$ be a $p$-tuple satisfying the system $S\left(D_{0}, \ldots, D_{p}\right)$. By definition, the $(p-1)$-tuple $\left(X_{0}, \ldots, X_{p-1}\right)$ satisfies the system $S\left(D_{0}, \ldots, D_{p-1}\right)$. This assumption yields

$$
\begin{aligned}
{\left[X_{0}, D_{0}\right] } & =\cdots=\left[X_{0}, D_{p-1}\right]=0, \\
{\left[X_{1}, D_{0}\right] } & =\cdots=\left[X_{1}, D_{p-2}\right]=0, \\
& \vdots \\
{\left[X_{p-1}, D_{0}\right] } & =0 .
\end{aligned}
$$

Writing the last equation of the system $S\left(D_{0}, \ldots, D_{p}\right)$ elementwise, we get

$$
X_{i j}^{(0)}\left(D_{i}^{(p)}-D_{j}^{(p)}\right)=-X_{i j}^{(1)}\left(D_{i}^{(p-1)}-D_{j}^{(p-1)}\right)-\cdots-X_{i j}^{(p)}\left(D_{i}^{(0)}-D_{j}^{(0)}\right) .
$$

If $(i, j) \notin \bigcap_{k=0}^{p-1} I\left(D_{k}\right)$, then $X_{i j}^{(0)}=0$ according to Lemma 3.3. If however $(i, j) \in \bigcap_{k=0}^{p-1} I\left(D_{k}\right)$ but $(i, j) \notin I\left(D_{p}\right)$, one has $D_{i}^{(k)}=D_{j}^{(k)}$ for $1 \leqslant k \leqslant p-1$ and $D_{i}^{(p)} \neq D_{j}^{(p)}$. Equation (4) then 


\section{E. Corel}

yields $X_{i j}^{(0)}=0$. We have thus

$$
(i, j) \notin I\left(D_{p}\right) \Longrightarrow X_{i j}^{(0)}=0,
$$

so the matrix $X_{0}$ commutes with $D_{p}$.

The matrices $\left(X_{1}, \ldots, X_{p}\right)$ then satisfy the $(p-1)$ th-order system $S\left(D_{0}, \ldots, D_{p-1}\right)$. According to the induction assumption, we get

$$
\begin{aligned}
{\left[X_{1}, D_{0}\right] } & =\cdots=\left[X_{1}, D_{p-1}\right]=0, \\
{\left[X_{2}, D_{0}\right] } & =\cdots=\left[X_{2}, D_{p-2}\right]=0, \\
& \vdots \\
{\left[X_{p-1}, D_{0}\right] } & =\left[X_{p-1}, D_{1}\right]=0, \\
{\left[X_{p}, D_{0}\right] } & =0,
\end{aligned}
$$

which proves the statement at order $p$.

Lemma 3.5. Let $B$ be a matrix of $\mathrm{M}_{n}(\mathbb{C})$ commuting with the matrices $D_{k}$ for all $k=0, \ldots, p$. Assume that there exists $p+1$ matrices $\left(X_{0}, \ldots, X_{p}\right)$ of $\mathrm{M}_{n}(\mathbb{C})$ such that

$$
\left[D_{0}, X_{0}\right]+\cdots+\left[D_{p}, X_{p}\right]=B
$$

Then $B=0$.

Proof. Written elementwise, the equation becomes

$$
X_{i j}^{(0)}\left(D_{i}^{(0)}-D_{j}^{(0)}\right)+\cdots+X_{i j}^{(p)}\left(D_{i}^{(p)}-D_{j}^{(p)}\right)=B_{i j} .
$$

If $(i, j) \in \bigcap_{k=1}^{p} I\left(D_{k}\right)$, we have $D_{i}^{(k)}=D_{j}^{(k)}$ for all $k=1, \ldots, p$, hence $B_{i j}=0$. If $(i, j) \notin \bigcap_{k=1}^{p} I\left(D_{k}\right)$, then $B_{i j}=0$, because $B$ commutes with all the matrices $D_{k}$. Therefore $B=0$.

Lemma 3.6. Assume that $(\varepsilon)$ and $(\tilde{\varepsilon})$ are two bases of $V$ in which the connection $\nabla$ has the same canonical form $B=D_{-m} t^{-m}+\cdots+D_{-1} t^{-1}+C$. The gauge matrix $P$ from $(\varepsilon)$ to $(\tilde{\varepsilon})$ then commutes with the irregular part of the canonical form $B$.

Proof. Assume that the gauge matrix $P$ from $(\varepsilon)$ to $(\tilde{\varepsilon})$ can be written as $P=t^{\nu} \hat{P}$, where

$$
\hat{P}=P_{0}+P_{1} t+\cdots+P_{k} t^{k}+\cdots \text {. }
$$

The gauge equation

$$
\theta_{t} \hat{P}-\nu \hat{P}=B \hat{P}-\hat{P} B
$$

gives rise to the following infinite system of matrix equations:

$$
\begin{gathered}
\sum_{t=0}^{k}\left[D_{-m+t}, P_{k-t}\right]=0(\text { in degree }-m+k) \text { for } 0 \leqslant k \leqslant m-1, \\
\sum_{t=0}^{m-1}\left[D_{-m+t}, P_{m+k-t}\right]=\left[P_{k}, C\right]+(k-\nu) P_{k}(\text { in degree } k) \text { for } k \geqslant 0,
\end{gathered}
$$




\section{ON FUCHS' RELATION FOR LINEAR DIFFERENTIAL SYSTEMS}

which we rewrite in expanded form as

$$
\begin{aligned}
{\left[D_{-m}, P_{0}\right] } & =0, \\
{\left[D_{-m}, P_{1}\right]+\left[D_{-m+1}, P_{0}\right] } & =0, \\
& \vdots \\
{\left[D_{-m}, P_{m-1}\right]+\cdots+\left[D_{-1}, P_{0}\right] } & =0, \\
{\left[D_{-m}, P_{m}\right]+\cdots+\left[D_{-1}, P_{1}\right] } & =\left[P_{0}, C\right]-\nu P_{0}, \\
& \vdots \\
{\left[D_{-m}, P_{m+k}\right]+\cdots+\left[D_{-1}, P_{k+1}\right] } & =\left[P_{k}, C\right]+(k-\nu) P_{k},
\end{aligned}
$$

With the notations used in Lemma 3.4 , the $(m-1)$-tuple $\left(P_{0}, \ldots, P_{m-1}\right)$ satisfies the system $S\left(D_{-m}, \ldots, D_{-1}\right)$. By means of Lemma 3.4 , we get

$$
\begin{aligned}
{\left[P_{0}, D_{-m}\right] } & =\cdots=\left[P_{0}, D_{-1}\right]=0, \\
{\left[P_{1}, D_{-m}\right] } & =\cdots=\left[P_{1}, D_{-2}\right]=0, \\
& \vdots \\
{\left[P_{m-2}, D_{-m}\right] } & =\left[P_{m-1}, D_{-m+1}\right]=0, \\
{\left[P_{m-1}, D_{-m}\right] } & =0 .
\end{aligned}
$$

Consider the system (6). Let us prove by induction on $k$ that if $\left(P_{0}, \ldots, P_{m-1}\right)$ satisfies the system (5), then $\left[P_{k}, C\right]+(k-\nu) P_{k}=0$ holds for any $k \geqslant 0$.

The matrices $P_{0}$ and $C$ commute with $D_{k}$ for all $k=-m, \ldots,-1$. According to Jacobi's identity, the same holds for $\left[P_{0}, C\right]$. Lemma 3.5 then yields $\left[P_{0}, C\right]-\nu P_{0}=0$. Assume now that the equation $\left[P_{t}, C\right]+(t-\nu) P_{t}=0$ holds for any $t<k$. Then for every $t=-1, \ldots, k-1$ the matrices $\left(P_{t+1}, \ldots, P_{m+t}\right)$ satisfy the equation

$$
\left[D_{-m}, P_{m+t}\right]+\cdots+\left[D_{-1}, P_{t+1}\right]=0 .
$$

We can put $D_{0}=\cdots=D_{k}=0$ because the matrix (0) is a diagonal matrix. The $(m+k)$-tuple $\left(P_{0}, \ldots, P_{m+k}\right)$ then satisfies the system $S\left(D_{-m}, \ldots, D_{k}\right)$, hence, according to Lemma 3.4 , we get

$$
\begin{aligned}
{\left[P_{0}, D_{-m}\right] } & =\cdots=\left[P_{0}, D_{k}\right]=0, \\
{\left[P_{1}, D_{-m}\right] } & =\cdots=\left[P_{1}, D_{k-1}\right]=0, \\
& \vdots \\
{\left[P_{m+k-1}, D_{-m}\right] } & =\left[P_{m+k}, D_{-m+1}\right]=0, \\
{\left[P_{m+k}, D_{-m}\right] } & =0 .
\end{aligned}
$$

In particular, $P_{k}$ commutes with $D_{-m}, \ldots, D_{0}$. Thus the same holds for the matrix $\left[P_{k}, B\right]+(k-\nu) P_{k}$. Therefore, we get $\left[P_{k}, B\right]+(k-\nu) P_{k}=0$. System (6) now becomes

$$
\sum_{t=0}^{m-1}\left[D_{-m+t}, P_{m+k-t}\right]=0, \quad \text { for } k \geqslant 0 .
$$

Hence the matrices $P_{k}$ commute with $D_{-m}, \ldots, D_{-1}$ for all $k \geqslant 0$. 


\section{E. Corel}

Proposition 3.4. Let $(\varepsilon)$ and $(\tilde{\varepsilon})$ be two bases of $V$ where the connection $\nabla$ has canonical forms

$$
\begin{aligned}
& B=D_{-m} t^{-m}+\cdots+D_{-1} t^{-1}+C \text { in }(\varepsilon), \\
& \tilde{B}=\tilde{D}_{-m} t^{-m}+\cdots+\tilde{D}_{-1} t^{-1}+\tilde{C} \text { in }(\tilde{\varepsilon}) .
\end{aligned}
$$

Let $P$ be the gauge matrix from $(\varepsilon)$ to $(\tilde{\varepsilon})$. Then the following equalities hold:

$$
\begin{aligned}
\tilde{D}_{-m} t^{-m}+\cdots+\tilde{D}_{-1} t^{-1} & =P^{-1}\left(D_{-m} t^{-m}+\cdots+D_{-1} t^{-1}\right) P, \\
\tilde{C} & =P^{-1} C P-P^{-1} \theta_{t} P .
\end{aligned}
$$

Proof. According to Proposition 3.2, there exists a matrix $T \in \mathrm{GL}_{n}(\mathbb{C})$ such that $T^{-1} C T=C^{\prime}$ and $T^{-1} D_{j} T=D_{j}^{\prime}$ for all $1 \leqslant j \leqslant s$. The gauge $\tilde{P}=P T$ preserves the matrix of the connection. Therefore it satisfies the gauge equation

$$
\theta_{t} \tilde{P}=B \tilde{P}-\tilde{P} B .
$$

Lemma 3.6 ensures that the matrix $\tilde{P}$ commutes with the irregular part $D_{-m} t^{-m}+\cdots+D_{-1} t^{-1}$. Hence,

$$
\begin{aligned}
D_{-m} t^{-m}+\cdots+D_{-1} t^{-1} & =\tilde{P}\left(D_{-m} t^{-m}+\cdots+D_{-1} t^{-1}\right) \tilde{P}^{-1} \\
& =P T\left(D_{-m} t^{-m}+\cdots+D_{-1} t^{-1}\right) T^{-1} P^{-1} \\
& =P\left(\tilde{D}_{-m} t^{-m}+\cdots+\tilde{D}_{-1} t^{-1}\right) P^{-1}
\end{aligned}
$$

and so (7) is established. Accordingly, we get

$$
\theta_{t} \tilde{P}=C \tilde{P}-\tilde{P} C
$$

which yields (8).

Corollary 3.2. If there exist two triples $\left(\nabla^{r}, \omega, \varepsilon\right)$ and $\left(\tilde{\nabla}^{r}, \tilde{\omega}, \tilde{\varepsilon}\right)$ satisfying the four conditions of Proposition 3.3, then one has $\nabla^{r}=\tilde{\nabla}^{r}$ and $\omega=\tilde{\omega}$.

Proof. The operator $\nabla_{\theta_{\zeta}}$ has canonical form $D_{-m} \zeta^{-m}+\cdots+D_{-1} \zeta^{-1}+C$ in the basis $(\varepsilon)$, and canonical form $\tilde{D}_{-m} \zeta^{-m}+\cdots+\tilde{D}_{-1} \zeta^{-1}+\tilde{C}$ in the basis $(\tilde{\varepsilon})$. Lemma 3.6, applied to $V_{H}$ equipped with the uniformizing parameter $\zeta$, shows that

$$
\operatorname{Mat}\left(\tilde{\nabla}_{\theta_{\zeta}}^{r},(\tilde{\varepsilon})\right)=\operatorname{Mat}\left(\nabla_{\theta_{\zeta}}^{r},(\varepsilon)\right)_{[P]}
$$

and

$$
\operatorname{Mat}(\tilde{\varphi},(\tilde{\varepsilon}))=P^{-1} \operatorname{Mat}(\varphi,(\varepsilon)) P,
$$

where $P$ is the gauge matrix from $(\varepsilon)$ to $(\tilde{\varepsilon})$. Hence we get $\nabla^{r}=\tilde{\nabla}^{r}$ and thus $\omega=\tilde{\omega}$.

We are now ready to prove Theorem 3 .

Proof of Theorem 3. We now show that the decomposition stated above is in fact defined on the base field $K$. Let $H$ be the field $K(\zeta)$. Let us denote with $\nabla_{H}^{r}$ the regular connection associated to $\nabla_{H}$, and let $\omega_{H}=\nabla_{H}-\nabla_{H}^{r}$. Let us choose a basis $(e)$ of $V$ over $K$, and put $\xi=e^{2 i \pi / p}$. The element $\sigma$ of the differential Galois group $\operatorname{Gal}(H / K)$ defined by putting $\sigma(\zeta)=\xi \zeta$ is a generator of the group. Choose a basis $\left(\zeta_{0}, \zeta_{1}, \ldots, \zeta_{p-1}\right)$ of $H$ over $K$ such that $\sigma\left(\zeta_{i}\right)=\zeta_{i+1} \bmod p$ holds. The family $\left(e_{i} \otimes \zeta_{j}\right)_{1 \leqslant i \leqslant n, 0 \leqslant j \leqslant p-1}$ is then a $K$-basis of $V_{H}$.

Denote with $\varphi_{i} \in(1 / \zeta) \mathbb{C}[1 / \zeta]$ the eigenvalues of $\left\langle\omega_{H}, \theta_{\zeta}\right\rangle$. There exists a basis $(\varepsilon)$ of $V_{H}$ such that:

i) $\operatorname{Mat}\left(\left(\nabla_{H}^{r}\right)_{\theta_{\zeta}},(\varepsilon)\right)=C \in \mathrm{M}_{n}(\mathbb{C})$;

ii) $\omega_{H}\left(\varepsilon_{i}\right)=\varphi_{i} \varepsilon_{i} d \zeta / \zeta$ for any $i=1, \ldots, n$. 


\section{ON FUCHS' RELATION FOR LINEAR DIFFERENTIAL SYSTEMS}

Consider the coordinate decomposition $\varepsilon_{i}=\sum_{j, k} U_{j k}^{i} e_{j} \otimes \zeta_{k}$ with $U_{j k}^{i} \in K$. The image of $\varepsilon_{i}$ under $\sigma$ is given by

$$
\begin{aligned}
\sigma\left(\varepsilon_{i}\right) & =\sum_{j, k} \sigma\left(U_{j k}^{i}\right) \sigma\left(e_{j}\right) \otimes \sigma\left(\zeta_{k}\right)=\sum_{j, k} U_{j k}^{i} e_{j} \otimes \zeta_{k+1 \bmod p} \\
& =\sum_{j, k} U_{j, k-1 \bmod p}^{i} e_{j} \otimes \zeta_{k} .
\end{aligned}
$$

The family $\left(\sigma\left(\varepsilon_{1}\right), \ldots, \sigma\left(\varepsilon_{n}\right)\right)$ is thus still a basis of $V_{H}$.

The map $\left(\nabla_{H}^{r}\right)^{\sigma}=\sigma \circ \nabla_{H}^{r} \circ \sigma^{-1}$ is $\mathbb{C}$-linear. For any $a \in H$ and any $v \in V_{H}$, the following holds:

$$
\begin{aligned}
\left(\nabla_{H}^{r}\right)^{\sigma}(a v) & =\sigma\left(\nabla_{H}^{r}\left(\sigma^{-1}(a v)\right)\right) \\
& =\sigma\left(\nabla_{H}^{r}\left(\sigma^{-1}(a)\right) \sigma^{-1}(v)\right) \\
& =\sigma\left(\sigma^{-1}(a) \nabla_{H}^{r}\left(\sigma^{-1}(v)\right)+\sigma^{-1} v \otimes d\left(\sigma^{-1}(a)\right)\right) \\
& =a \sigma\left(\nabla_{H}^{r}\left(\sigma^{-1}(v)\right)\right)+v \otimes \sigma\left(d\left(\sigma^{-1}(a)\right)\right) .
\end{aligned}
$$

Since $\sigma$ is a differential automorphism of $H$, it commutes with the differential $d$, hence

$$
\left(\nabla_{H}^{r}\right)^{\sigma}(a v)=a\left(\nabla_{H}^{r}\right)^{\sigma}(v)+v \otimes d a,
$$

so $\left(\nabla_{H}^{r}\right)^{\sigma}$ is indeed a connection on $V_{H}$. In the basis $(\sigma(\varepsilon))$, we have

$$
\begin{aligned}
\left(\nabla_{H}^{r}\right)^{\sigma}\left(\sigma\left(\varepsilon_{i}\right)\right)=\sigma \circ \nabla_{H}^{r} \circ \sigma^{-1}\left(\sigma\left(\varepsilon_{i}\right)\right) & =\sigma\left(\nabla_{H}^{r}\left(\varepsilon_{i}\right)\right) \\
& =\sigma\left(\sum_{j=1}^{n} C_{j i} \varepsilon_{j}\right) \\
& =\sum_{j=1}^{n} \sigma\left(C_{j i}\right) \sigma\left(\varepsilon_{j}\right) \\
& =\sum_{j=1}^{n} C_{j i} \sigma\left(\varepsilon_{j}\right) .
\end{aligned}
$$

The connection $\nabla_{H}^{r}$ has a simple pole in the basis $(\sigma(\varepsilon))$; thus it is a regular connection.

The map $\omega_{H}^{\sigma}=\sigma \circ \omega_{H} \circ \sigma^{-1}$ is $\mathbb{C}$-linear. For any $a \in H$ and any $v \in V_{H}$, the following holds:

$$
\begin{aligned}
\omega_{H}^{\sigma}(a v) & =\sigma\left(\omega_{H}\left(\sigma^{-1}(a v)\right)\right) \\
& =\sigma\left(\omega_{H}\left(\sigma^{-1}(a) \sigma^{-1}(v)\right)\right) \\
& =\sigma\left(\sigma^{-1}(a) \omega_{H}\left(\sigma^{-1}(v)\right)\right) \\
& =a \omega_{H}^{\sigma}(v) .
\end{aligned}
$$

Therefore $\omega_{H}^{\sigma}$ is $H$-linear. On the other hand, we have

$$
\begin{aligned}
\omega_{H}^{\sigma}\left(\sigma\left(\varepsilon_{i}\right)\right)=\sigma \circ \omega_{H} \circ \sigma^{-1}\left(\sigma\left(\varepsilon_{i}\right)\right) & =\sigma\left(\omega_{H}\left(\varepsilon_{i}\right)\right) \\
& =\sigma\left(\varphi_{i} \varepsilon_{i} \frac{d \zeta}{\zeta}\right) \\
& =\sigma\left(\varphi_{i}\right) \sigma\left(\varepsilon_{i}\right) \sigma\left(\frac{d \zeta}{\zeta}\right) .
\end{aligned}
$$

Since the map $\sigma$ is an element of the Galois group, we get

$$
\sigma\left(\frac{d \zeta}{\zeta}\right)=\frac{d(\sigma(\zeta))}{\sigma \zeta}=\frac{d(\xi \zeta)}{\xi \zeta}=\frac{d \zeta}{\zeta}
$$




\section{E. Corel}

Accordingly,

$$
\omega_{H}^{\sigma}\left(\sigma\left(\varepsilon_{i}\right)\right)=\sigma\left(\varphi_{i}\right) \sigma\left(\varepsilon_{i}\right) \frac{d \zeta}{\zeta}
$$

holds. The collection of the vectors $\sigma\left(\varepsilon_{i}\right)$ forms a basis of eigenvectors of $\left\langle\omega_{H}^{\sigma}, \theta_{\zeta}\right\rangle$. The eigenvalues of $\left\langle\omega_{H}^{\sigma}, \theta_{\zeta}\right\rangle$ are the images $\sigma\left(\varphi_{i}\right)$ of the $\varphi_{i}$ who also belong to $(1 / \zeta) \mathbb{C}[1 / \zeta]$.

The connection $\nabla$ is defined over $K$. It is thus invariant under the action of the Galois group $\operatorname{Gal}(H / K)$ and so $\nabla_{H}$ satisfies

$$
\nabla_{H}=\left(\nabla_{H}\right)^{\sigma}=\left(\nabla_{H}^{r}\right)^{\sigma}+\omega_{H}^{\sigma} .
$$

The connection $\left(\nabla_{H}^{r}\right)^{\sigma}$ and the map $\omega_{H}^{\sigma}$ satisfy the conditions of Proposition 3.3 in the basis $\left(\sigma\left(\varepsilon_{1}\right), \ldots, \sigma\left(\varepsilon_{n}\right)\right)$. The uniqueness of the decomposition implies that $\left(\nabla_{H}^{r}\right)^{\sigma}=\nabla_{H}^{r}$ and thus $\sigma \circ \nabla_{H}^{r}=\nabla_{H}^{r} \circ \sigma$ hold. Hence there exists a regular connection $\nabla^{r}$ on $V$ satisfying the assumptions of Theorem 3, such that $\nabla_{H}^{r}=\nabla^{r} \otimes 1_{H}+\mathrm{id}_{V} \otimes d$. This connection is unique. The map $\omega=\nabla-\nabla^{r}$ satisfying $\omega \otimes 1=\omega_{H}$ is what we called the determinant map.

\section{Levelt lattices and exponents}

\subsection{The unramified case}

Let $(V, \nabla)$ be a finite-dimensional $K$-vector space endowed with a connection. Let $\nabla=\nabla^{r}+\omega$ be the canonical decomposition of $\nabla$. Assume in this subsection that the determinant endomorphism $\varphi$ of $\nabla$ has its eigenvalues in $(1 / z) \mathbb{C}[1 / z]$. We will then say that $\nabla$ is unramified. Denote the attached direct sum with $V=\bigoplus_{i=1}^{s} V_{i}$. Let us consider a lattice $\Lambda$ of $V$.

Definition 13. A lattice is said to be compatible with the connection $\nabla$ if it is stable under $\nabla_{\theta}^{r}$ and compatible with the direct sum $\bigoplus_{i=1}^{s} V_{i}$ attached to $\nabla$.

Proposition 4.1. The set of sublattices of $\Lambda$ which are compatible with the connection $\nabla$ has a unique maximal element.

Proof. The connection $\nabla^{r}$ is regular. Thus there exists a $\nabla_{\theta}^{r}$-stable lattice $M$ of $V$. After Corollary 2.2 , there exists a largest $\nabla_{\theta}^{r}$-stable sublattice $N$ of $\Lambda$. Since the direct sum $\bigoplus_{i=1}^{s} V_{i}$ is stable under the action of $\nabla_{\theta}^{r}$, the lattice $\bigoplus_{i=1}^{s} N \cap V_{i}$ is the largest sublattice of $\Lambda$ compatible with $\nabla$.

Definition 14. Let $\Lambda$ be a lattice of $V$. We call Levelt lattice for the connection $\nabla$ attached to the lattice $\Lambda$ the largest sublattice $\Lambda_{L}(\nabla)$ of $\Lambda$ compatible with the connection $\nabla$.

Definition 15. We call exponents of the connection $\nabla$ attached to the lattice $\Lambda$ the eigenvalues $\left(e_{i}^{\Lambda}(\nabla)\right)_{i=1, \ldots, n}$ of the residue of the associated regular connection $\nabla^{r}$ with respect to the lattice $\Lambda_{L}(\nabla)$. We denote with $N_{i}^{\Lambda}(\nabla)$ the integer part of the real part of the exponents $e_{i}^{\Lambda}(\nabla)$, and call them valuations of the connection $\nabla$ attached to the lattice $\Lambda$.

We sometimes write $e_{i}^{\Lambda}(\nabla)=N_{i}^{\Lambda}(\nabla)+\tilde{e}_{i}^{\Lambda}(\nabla)$. If so, we will call $\tilde{e}_{i}^{\Lambda}(\nabla)$ the non-integer or invariant part of $e_{i}^{\Lambda}(\nabla)$.

Remark 7. These two definitions extend previous notions that we defined in the regular case [Cor01a]. The exponents in the sense of Definition 15 extend the notion of exponents defined by Levelt [Lev61] for analytic systems at a regular singularity.

The definition of the Levelt lattice easily yields the following result. 


\section{ON FUCHS' RELATION FOR LINEAR DIFFERENTIAL SYSTEMS}

Lemma 4.1. Let $\Lambda$ be a lattice of $V$.

i) If $\tilde{\Lambda} \subset \Lambda$ is a sublattice of $\Lambda$, then $\tilde{\Lambda}_{L} \subset \Lambda_{L}$ holds.

ii) Let $\Lambda_{1}$ and $\Lambda_{2}$ be two free $\mathcal{O}$-submodules of $V$ such that $\Lambda=\Lambda_{1} \oplus \Lambda_{2}$. If the $K$-vector spaces $V_{1}=\Lambda_{1} \otimes_{\mathcal{O}} K$ and $V_{2}=\Lambda_{2} \otimes_{\mathcal{O}} K$ are stable under $\nabla_{\theta}$, then the Levelt lattice $\Lambda_{L}$ of $\Lambda$ satisfies

$$
\Lambda_{L}=\left(\Lambda_{1}\right)_{L} \oplus\left(\Lambda_{2}\right)_{L}
$$

Lemma 4.2. Let $(V, \nabla)$ be a vector space endowed with a connection and let $P \in(1 / z) \mathbb{C}[1 / z]$. The map $\nabla+P \mathrm{id}_{V} \otimes d z / z$ is a connection on $V$, and

$$
\Lambda_{L}\left(\nabla+P \operatorname{id}_{V} \otimes \frac{d z}{z}\right)=\Lambda_{L}(\nabla) \text { holds for any lattice } \Lambda \text { of } V .
$$

Proof. If $\nabla=\nabla^{r}+\omega$ is the canonical decomposition of $\nabla$, then

$$
\nabla+P \operatorname{id}_{V} \otimes \frac{d z}{z}=\nabla^{r}+\left(\omega+P \operatorname{id}_{V} \otimes \frac{d z}{z}\right)
$$

is the corresponding canonical decomposition of $\nabla+P \operatorname{id}_{V} \otimes d z / z$.

Lemma 4.3. If the connection $\nabla$ has only one determinant factor, then the following hold, for any lattice $\Lambda$ of $V$ :

i) $\Lambda_{L}(\nabla)=\Lambda_{L}\left(\nabla^{r}\right)$;

ii) $0 \leqslant \mathfrak{p}_{\Lambda}\left(\nabla^{r}\right) \leqslant \mathfrak{p}_{\Lambda}(\nabla)$.

Proof. Let $\varphi=f \mathrm{id}_{V}$ be the determinant map of $\nabla$. Statement i is a straightforward consequence of Lemma 4.2. Take $v \in \Lambda$. We have

$$
\begin{aligned}
v_{\Lambda}\left(\nabla_{\theta}^{r}(v)\right)=v_{\Lambda}\left(\nabla_{\theta}(v)-f v\right) & \geqslant \min \left(v_{\Lambda}\left(\nabla_{\theta}(v)\right), v_{\Lambda}(f v)\right) \\
& \geqslant \min \left(v_{\Lambda}\left(\nabla_{\theta}(v)\right), v(f)+v_{\Lambda}(v)\right) .
\end{aligned}
$$

Since $v_{\Lambda}\left(\nabla_{\theta}^{r}(\Lambda)\right)=\inf _{v \in \Lambda} v_{\Lambda}\left(\nabla_{\theta}^{r}(v)\right)$, we find that

$$
v_{\Lambda}\left(\nabla_{\theta}^{r}(\Lambda)\right) \geqslant \min \left(v_{\Lambda}\left(\nabla_{\theta}(\Lambda)\right), v(f)\right) .
$$

But $-v(f)=\kappa(\nabla) \leqslant \mathfrak{p}_{\Lambda}(\nabla)$ holds by definition. Thus we get

$$
-v_{\Lambda}\left(\nabla_{\theta}^{r}(\Lambda)\right) \leqslant-v_{\Lambda}\left(\nabla_{\theta}(\Lambda)\right)
$$

and statement ii follows.

\subsection{The ramified case}

Assume here that the $K$-vector space endowed with a connection $(V, \nabla)$ has ramification order $p>0$. Let us take the notations of $\S 3.1$. Denote with $H=K[T] /\left(T^{p}-z\right)$ the minimal ramification extension, with $\mathcal{O}_{H}$ the corresponding valuation ring, with $V_{H}=V \otimes_{K} H$ the vector space obtained under extension of scalars and with $\nabla_{H}$ the unique extension of the connection $\nabla$. Let $\nabla=\nabla^{r}+\omega$ be the canonical decomposition of $\nabla$, and $V_{H}=\bigoplus_{i=1}^{s} V_{i}$ the attached direct sum.

Let $\Lambda$ be a lattice of $V$, and $\Lambda_{H}=\Lambda \otimes_{\mathcal{O}} \mathcal{O}_{H}$. Choose a $p$ th root $\zeta$ of $z$, and denote with $\theta_{\zeta}$ the derivation $\zeta d / d \zeta$.

LEMma 4.4. Under the former assumptions, the following hold:

i) The $\operatorname{sum} \nabla_{H}=\left(\nabla^{r}\right)_{H}+\omega \otimes 1$ is the canonical decomposition of $\nabla_{H}$.

ii) The connection $\nabla_{H}$ is unramified (with respect to $\zeta$ ). 


\section{E. Corel}

Definition 16. Let $\Lambda$ be a lattice of $V$.

1) We call Levelt lattice for the connection $\nabla$ attached to the lattice $\Lambda$ the Levelt lattice attached to the lattice $\Lambda_{H}$ for the connection $\nabla_{H}$. One denotes it with $\Lambda_{L}(\nabla)$, although usually it is not defined over $\mathcal{O}$.

2) We call exponents of the connection $\nabla$ attached to the lattice $\Lambda$ the eigenvalues $\left(e_{i}^{\Lambda}(\nabla)\right)_{i=1, \ldots, n}$ of the compatible residue $\operatorname{Res}_{\Lambda_{L}(\nabla)}^{c} \nabla_{H}^{r}$ of the regular connection $\nabla_{H}^{r}$ attached to $\nabla_{H}$ with respect to the lattice $\Lambda_{L}(\nabla)$.

Lemma 4.5. The Levelt lattice $\Lambda_{L}(\nabla)$ is independent of the choice of the uniformizing parameter $\zeta$.

Proof. Let us consider an automorphism $\sigma \in \mathrm{Gal}(\mathrm{H} / \mathrm{K})$ acting on $V_{H}$ as in the proof of Theorem 3. The lattice $\sigma\left(\Lambda_{L}(\nabla)\right)$ is still $\left(\nabla_{\theta}^{r}\right)_{H}$-stable, and it is compatible with the attached direct $\operatorname{sum} \bigoplus_{i=1}^{s} V_{i}$. We have

$$
\sigma\left(\Lambda_{L}(\nabla)\right) \subset \sigma\left(\Lambda_{H}\right)=\Lambda_{H},
$$

because $\Lambda_{H}$ is the tensor extension of a lattice of $V$. Hence $\sigma\left(\Lambda_{L}(\nabla)\right) \subset \Lambda_{L}(\nabla)$. The former reasoning also applies to $\sigma^{-1}$, thus $\sigma\left(\Lambda_{L}(\nabla)\right)=\Lambda_{L}(\nabla)$. Therefore we proved that $\Lambda_{L}(\nabla)$ is independent of the choice of $\zeta$.

Note that any ramification of order $p^{\prime}$ divisible by $p$ gives with this definition the same set of exponents.

\subsection{The Katz lattice}

Assume in this subsection that the connection is unramified. The Katz rank $\kappa(\nabla)$ of $\nabla$ is then equal to the minimal Poincaré rank of $\nabla$ on all lattices of $V$, that we called the order of the singularity $m(\nabla)$. By means of Corollary 2.2 the following definition makes sense.

Definition 17. We call the Katz lattice of $\nabla$ attached to the lattice $\Lambda$ the largest sublattice $\Lambda_{K}(\nabla)$ of $\Lambda$ of minimal Poincaré rank.

Lemma 4.6. Let $\Lambda$ be a lattice of $V$.

i) If the connection $\nabla$ is regular, then $\Lambda_{K}(\nabla)=\Lambda_{L}(\nabla)$ holds.

ii) If the polar map $\bar{\nabla}^{\Lambda}$ of the connection $\nabla$ is non-nilpotent, then the Katz lattice $\Lambda_{K}(\nabla)$ of $\Lambda$ satisfies

$$
\Lambda_{K}(\nabla)=\Lambda
$$

iii) The polar map $\bar{\nabla}^{\Lambda_{K}(\nabla)}$ is non-nilpotent.

Proof. The Katz rank of a regular connection is zero. The definitions of the Katz lattice and of the Levelt lattice then coincide. In the unramified case, the map $\bar{\nabla}^{\Lambda}$ is non-nilpotent only if there exists a determinant factor of degree equal to the Poincaré rank. Therefore condition iii holds. In this case, $\mathfrak{p}_{\Lambda}(\nabla)=\kappa(\nabla)$ also holds, hence $\Lambda_{K}(\nabla)=\Lambda$.

Proposition 4.2. Let $\Lambda$ be a lattice of $V$. Let $\Lambda_{K}=\Lambda_{K}(\nabla)$ be the attached Katz lattice and $\mathcal{E}_{\Lambda}\left(\Lambda_{K}\right)=\left(k_{1}, \ldots, k_{n}\right)$ the sequence of its elementary divisors in $\Lambda$. We denote with $\mathfrak{p}=\mathfrak{p}_{\Lambda}(\nabla)$ the Poincaré rank of $\nabla$ on $\Lambda$, and with $\kappa=\kappa(\nabla)$ the Katz rank of $\nabla$. Then the following inequalities hold:

$$
\max _{i=1, \ldots, n-1} k_{i+i}-k_{i} \leqslant \mathfrak{p}-\kappa \leqslant k_{n}
$$




\section{ON FUCHS' RELATION FOR LINEAR DIFFERENTIAL SYSTEMS}

Proof. By definition, one has $\kappa \leqslant \mathfrak{p}$. If $\mathfrak{p}=0$, the connection is regular: in this case $\Lambda_{K}=\Lambda_{L}=\Lambda$, and thus $k_{1}=\cdots=k_{n}=0$. Assume in the sequel that $\mathfrak{p}>0$.

Let $(\varepsilon)$ be a Smith basis of $\Lambda$ for $\Lambda_{K}$. We denote with $X=\left(x_{1}, \ldots, x_{n}\right)$ an $n$-tuple of integers, with $z^{X}$ the matrix

$$
z^{X}=\left(\begin{array}{ccc}
z^{x_{1}} & & 0 \\
& \ddots & \\
0 & & z^{x_{n}}
\end{array}\right)
$$

and with $\left(z^{X} \varepsilon\right)$ the family $\left(z^{x_{1}} \varepsilon_{1}, \ldots, z^{x_{n}} \varepsilon_{n}\right)$. Denoting with $A=\operatorname{Mat}\left(\nabla_{\theta},(\varepsilon)\right)$ the matrix of the connection in the basis $(\varepsilon)$ we have

$$
\operatorname{Mat}\left(\nabla_{\theta},\left(z^{X} \varepsilon\right)\right)=A_{\left[z^{X}\right]}=\left(A_{i j} z^{x_{j}-x_{i}}-\delta_{i, j} x_{i}\right)_{1 \leqslant i, j \leqslant n} .
$$

The Katz lattice $\Lambda_{K}$ has Poincaré rank $\kappa$. Call $\mathcal{E}$ the sequence $\left(k_{1}, \ldots, k_{n}\right)$. The matrix $A_{\left[z^{\mathcal{E}}\right]}$ then has its coefficients in $z^{-\kappa} \mathcal{O}$. Therefore,

$$
v\left(A_{i j}\right)-k_{i}+k_{j} \geqslant-\kappa \text { for all } 1 \leqslant i, j \leqslant n .
$$

Since $\mathfrak{p}=\max _{1 \leqslant i, j \leqslant n}\left(-v\left(A_{i j}\right)\right)$, the right-hand side of the proposition

$$
\mathfrak{p}-\kappa \leqslant \max _{1 \leqslant i, j \leqslant n}\left(k_{j}-k_{i}\right)=k_{n}
$$

follows. On the other hand, the index $\left[\Lambda: \Lambda_{K}\right]=\sum_{i=1}^{n} k_{i}$ is minimal among the indexes in $\Lambda$ of all sublattices of $\Lambda$ of minimal Poincaré rank. For any $T=\left(t_{1}, \ldots, t_{n}\right) \in \mathbb{Z}^{n}$ such that $0 \leqslant t_{1} \leqslant \cdots \leqslant t_{n}$ and $\sum_{i=1}^{n} t_{i}<\sum_{i=1}^{n} k_{i}$, the lattice spanned by $\left(z^{T} \varepsilon\right)$ has strictly larger Poincaré rank than $\kappa$. There exists thus a couple of indexes $\left(i_{(T)}, j_{(T)}\right) \in\{1, \ldots, n\}^{2}$ such that

$$
v\left(A_{i_{(T)} j_{(T)}}\right)-t_{i_{(T)}}+t_{j_{(T)}}<-\kappa .
$$

Let $\ell$ be an index such that $k_{\ell+1} \geqslant 1$. Let us show that $k_{\ell+1}-k_{\ell} \leqslant \mathfrak{p}-\kappa$. Let $t_{i}=k_{i}$ for $i \leqslant \ell$ and $t_{i}=k_{i}-1$ for $i \geqslant \ell+1$. Then there exists a pair $(i, j)$ and $\varepsilon=-1,0$ or 1 such that

$$
-\kappa>v\left(A_{i j}\right)-t_{i}+t_{j}=v\left(A_{i j}\right)-k_{i}+k_{j}+\varepsilon \geqslant \varepsilon-\kappa .
$$

Hence $v\left(A_{i j}\right)=k_{i}-k_{j}$ and $i \leqslant \ell \leqslant \ell+1 \leqslant j$, and so

$$
k_{\ell+1}-k_{\ell} \leqslant k_{j}-k_{i}=-v\left(A_{i j}\right) \leqslant \mathfrak{p}-\kappa .
$$

The left-hand side

$$
\max _{i=1, \ldots, n-1} k_{i+1}-k_{i} \leqslant \mathfrak{p}-\kappa
$$

follows.

Corollary 4.1. Let $\Lambda$ be a lattice of $V$. Let $\mathfrak{p}=\mathfrak{p}_{\Lambda}(\nabla)$ be the Poincaré rank on the lattice $\Lambda$ and $\kappa=\kappa(\nabla)$ be the Katz rank of the connection. The index of the Katz lattice $\Lambda_{K}$ in $\Lambda$ satisfies

$$
\mathfrak{p}-\kappa \leqslant\left[\Lambda: \Lambda_{K}\right] \leqslant \frac{n(n-1)}{2}(\mathfrak{p}-\kappa) .
$$

Proof. The estimate follows from Proposition 4.2, since $\left[\Lambda: \Lambda_{K}\right]=\sum_{i=1}^{n} k_{i}$ holds.

Note that Corollary 4.1 yields the following result, which we stated for the regular case in [Cor99a].

Corollary 4.2. If the connection $\nabla$ is regular, then for any lattice $\Lambda$ of $V$, the index of its Levelt lattice satisfies

$$
\mathfrak{p}_{\Lambda}(\nabla) \leqslant\left[\Lambda: \Lambda_{L}\right] \leqslant \frac{n(n-1)}{2} \mathfrak{p}_{\Lambda}(\nabla)
$$




\section{E. COREL}

Proof. Indeed, in this case the Katz rank is zero, and the Katz lattice is equal to the Levelt lattice.

Lemma 4.7. Let $\Lambda$ be a lattice of $V$. The Katz lattice $\Lambda_{K}=\Lambda_{K}(\nabla)$ and the Levelt lattice

$$
\Lambda_{L}=\Lambda_{L}(\nabla)
$$

attached to $\Lambda$ satisfy

$$
\left(\Lambda_{K}\right)_{L}(\nabla)=\Lambda_{L}
$$

Proof. The Poincaré rank on the Levelt lattice $\Lambda_{L}$ is equal to the Katz rank of the connection $\nabla$. Therefore, $\Lambda_{L} \subset \Lambda_{K}$. Since $\Lambda_{L}$ is compatible with $\nabla$, it follows that $\Lambda_{L} \subset\left(\Lambda_{K}\right)_{L}(\nabla)$. There is no strictly larger lattice compatible with $\nabla$ than $\Lambda_{L}$. However $\Lambda_{K}$ is a sublattice of $\Lambda$ compatible with $\nabla$, whence $\left(\Lambda_{K}\right)_{L}(\nabla)=\Lambda_{L}$.

\subsection{Duality and special lattices}

Let us now consider the dual connection $\nabla^{*}$ induced by $\nabla$ on the $K$-dual $V^{*}$ of $V$.

Let $M$ be a lattice of $V$ spanned over $\mathcal{O}$ by a basis $(e)$ of $V$ and let $\left(e^{*}\right)$ be the dual basis of $(e)$. Lattices are well behaved towards duality, i.e. one has

$$
\operatorname{Hom}_{K}(M, \mathcal{O})=\operatorname{Hom}_{\mathcal{O}}(M, \mathcal{O})=\mathcal{L}\left(e^{*}\right)
$$

(cf. [Bou85, Part VII, $\S 4$, no. 2, p. 243]). We denote with $M^{*}$ the dual lattice $\operatorname{Hom}_{\mathcal{O}}(M, \mathcal{O})$ of $M$. The Poincaré rank of the dual connection $\nabla^{*}$ on the dual lattice $M^{*}$ satisfies $\mathfrak{p}_{M^{*}}\left(\nabla^{*}\right)=\mathfrak{p}_{M}(\nabla)$. In a similar way as for Corollary 2.2, we have the following result.

Lemma 4.8. Let $\nabla$ be a connection on $V$ of order of singularity $m=m(\nabla)$. Then, for any $k \geqslant m$, and any lattice $\Lambda$ of $V$, there exists a unique minimal lattice $\Lambda^{k}$ containing $\Lambda$ such that $\mathfrak{p}_{\Lambda^{k}}(\nabla) \leqslant k$.

Since $M$ is a sublattice of $\Lambda$ implies that $M^{*} \supset \Lambda^{*}$, Remark 5 yields the following result.

Corollary 4.3. Let $\nabla$ be a connection on $V$. Let $m$ be its order of singularity. Then, for any $k \geqslant m$, and any lattice $\Lambda$ of $V$, the saturated lattice $\mathcal{F}_{z^{k} \theta}^{n-1}\left(\Lambda^{*}\right)$ of the dual lattice $\Lambda^{*}$ with respect to the dual connection $\nabla^{*}$ satisfies

$$
\mathcal{F}_{z^{k} \theta}^{n-1}\left(\Lambda^{*}\right)^{*}=\Lambda_{k}(\nabla)
$$

Remark 8. Since $\Lambda_{L}(\nabla)=\Lambda_{0}$ when $\nabla$ is regular, this result gives rise to an algorithm that computes the Levelt lattice in the regular case, which differs from the algorithm given by Levelt [Lev01]. When the connection is unramified, we get an algorithm to compute the Katz lattice, since in that case one has $\Lambda_{K}(\nabla)=\Lambda_{m}$, if we denote with $m$ the order of singularity of $\nabla$. We shall give the corresponding algorithm in the appendix.

\section{Fuchs' relation}

In this section, we prove the results yielding the generalization of Fuchs' relation. We shall need the following classical result.

\subsection{Sibuya's lemma}

Sibuya's lemma (cf. [Lev75, p. 10]) is a fundamental result for formal reduction algorithms at an irregular singularity. 


\section{ON FUCHS' RELATION FOR LINEAR DIFFERENTIAL SYSTEMS}

Lemma 5.1. Let $\Lambda$ be a lattice of $V$, let $\mathfrak{p}=\mathfrak{p}_{\Lambda}(\nabla)>0$ be the Poincaré rank of the connection $\nabla$ on $\Lambda$. Let $\pi$ be the canonical projection of $\Lambda$ on $\bar{\Lambda}=\Lambda / z \Lambda$ and let $\bar{\nabla}^{\Lambda}$ be the induced polar map on $\bar{\Lambda}=\Lambda / z \Lambda$. Assume that there exist two $\mathbb{C}$-vector subspaces $F_{1}$ and $F_{2}$ of $\bar{\Lambda}$ such that the following conditions hold:

i) $\bar{\Lambda}=F_{1} \oplus F_{2}$;

ii) $F_{1}$ and $F_{2}$ are stable under $\bar{\nabla}^{\Lambda}$;

iii) the restrictions $\bar{\nabla}_{1}=\bar{\nabla}_{\mid F_{1}}^{\Lambda}$ and $\bar{\nabla}_{2}=\bar{\nabla}_{\mid F_{2}}^{\Lambda}$ have no eigenvalue in common.

Then there exist two unique free $z^{\mathfrak{p}} \nabla_{\theta}$-stable $\mathcal{O}$-submodules $\Lambda_{1}$ and $\Lambda_{2}$ of $\Lambda$ satisfying:

1) $\Lambda=\Lambda_{1} \oplus \Lambda_{2}$;

2) $F_{1}=\pi\left(\Lambda_{1}\right)$ and $F_{2}=\pi\left(\Lambda_{2}\right)$.

\subsection{Estimates for lattice invariants}

Proposition 5.1. Let $\nabla$ be an unramified connection on $V$ and $\Lambda$ be a lattice of $V$. Let $\mathfrak{p}=\mathfrak{p}_{\Lambda}(\nabla)$ be the Poincaré rank, and $\kappa=\kappa(\nabla)$ be the Katz rank of $\nabla$ on $\Lambda$. The Levelt lattice $\Lambda_{L}(\nabla)$ of $\Lambda$ satisfies the inequalities

$$
\mathfrak{p}-\kappa \leqslant\left[\Lambda: \Lambda_{L}(\nabla)\right] \leqslant \frac{n(n-1)}{2} \mathfrak{p}-\frac{1}{2} \operatorname{irr}(\text { End } \nabla),
$$

where $\operatorname{irr}($ End $\nabla)$ denotes the Malgrange irregularity of the connection End $\nabla$ induced by $\nabla$ on End $V$.

Recall that, if the vector space endowed with a connection $(V, \nabla)$ has determinant factors $Q_{i}$ with multiplicity $n_{i}$, the Malgrange irregularity index of End $\nabla$ is equal to

$$
\operatorname{irr}(\text { End } \nabla)=-2 \sum_{1 \leqslant i<j \leqslant s} n_{i} n_{j} v\left(Q_{i}-Q_{j}\right)=-2 \sum_{1 \leqslant i<j \leqslant s} n_{i} n_{j} v\left(\varphi_{i}-\varphi_{j}\right)
$$

(cf. [Ber98, p. 10]). The following lemma will be of use in the proof.

Lemma 5.2. Let $m \leqslant n$ two integers. The equality

$$
\frac{m(m-1)}{2}+\frac{(n-m)(n-m-1)}{2}=\frac{n(n-1)}{2}-m(n-m)
$$

holds.

Proof of Proposition 5.1. Corollary 4.1 yields

$$
\mathfrak{p}-\kappa \leqslant\left[\Lambda: \Lambda_{K}(\nabla)\right] \leqslant \frac{n(n-1)}{2}(\mathfrak{p}-\kappa) .
$$

Since we have

$$
\left[\Lambda: \Lambda_{L}(\nabla)\right]=\left[\Lambda: \Lambda_{K}(\nabla)\right]+\left[\Lambda_{K}(\nabla): \Lambda_{L}(\nabla)\right]
$$

it is enough to estimate the index $\left[\Lambda_{K}(\nabla): \Lambda_{L}(\nabla)\right]$. We use induction on the number $s$ of distinct determinant factors of $\nabla$.

Assume that the connection has only one determinant factor, denoted with $Q$. Then $\kappa=-v(Q)$ and $\operatorname{irr}($ End $\nabla)=0$ hold. According to Lemma 4.3, part i, the Levelt lattice of $\Lambda$ satisfies the equality $\Lambda_{L}(\nabla)=\Lambda_{L}\left(\nabla^{r}\right)$. By means of Lemma 4.7 we get $\Lambda_{L}(\nabla)=\left(\Lambda_{K}(\nabla)\right)_{L}\left(\nabla^{r}\right)$. The connection $\nabla^{r}$ is regular and its Poincaré rank $\tilde{\mathfrak{p}}=\mathfrak{p}_{\Lambda_{K}(\nabla)}\left(\nabla^{r}\right)$ on the Katz lattice $\Lambda_{K}(\nabla)$ satisfies

$$
0 \leqslant \tilde{\mathfrak{p}} \leqslant \kappa .
$$




\section{E. Corel}

After Corollary 4.2, the inequalities $0 \leqslant\left[\Lambda_{K}(\nabla): \Lambda_{L}(\nabla)\right] \leqslant \frac{1}{2} n(n-1) \kappa$ hold. Hence we get

$$
\mathfrak{p}-\kappa \leqslant\left[\Lambda: \Lambda_{L}(\nabla)\right] \leqslant \frac{n(n-1)}{2}(\mathfrak{p}-\kappa)+\frac{n(n-1)}{2} \kappa=\frac{n(n-1)}{2} \mathfrak{p} .
$$

The statement for $s=1$ follows, since in that case $\operatorname{irr}($ End $\nabla)=0$.

Let $s \geqslant 2$ be an integer. Assume that

$$
0 \leqslant\left[\Lambda_{K}(\nabla): \Lambda_{L}(\nabla)\right] \leqslant \frac{n(n-1)}{2} \kappa-\frac{1}{2} \operatorname{irr}(\text { End } \nabla)
$$

holds for any $t<s$, for any vector space endowed with a connection $(V, \nabla)$ having $t$ distinct determinant factors, and for any lattice $\Lambda$ of $V$.

Let $\left(\varphi_{i}\right)_{i=1, \ldots, s}$ be the distinct determinant factors of $(V, \nabla)$. The valuation of every $\varphi_{i}$ is negative. Assume the $\left(\varphi_{i}\right)$ arranged by increasing valuation. Then $v\left(\varphi_{1}\right)=-\kappa$ holds. We shall say that $\varphi_{i}$ and $\varphi_{j}$ are equivalent up to order $k$ if $v\left(\varphi_{i}-\varphi_{j}\right) \geqslant-\kappa+k+1$. Let $\Lambda$ be a lattice of $V$. Let us consider the Katz lattice $\Lambda_{K}(\nabla)$. The eigenvalues of the polar map $\bar{\nabla}^{\Lambda_{K}(\nabla)}$ are equal to the coefficients of valuation $-\kappa$ of the attached eigenvalues $\varphi_{i}=\theta Q_{i}$. Two situations may occur.

a) The polar map $\bar{\nabla}^{\Lambda_{K}(\nabla)}$ has at least two distinct eigenvalues. If so, one of them is not zero. Let us call $W$ the eigenspace of $\Lambda / z \Lambda$ corresponding to a non-zero eigenvalue of $\bar{\nabla}^{\Lambda_{K}(\nabla)}$. Sibuya's lemma ensures then that there exist two free $\mathcal{O}_{\text {-submodules }} \Lambda_{1}$ (whose image in $\Lambda / z \Lambda$ is $W$ ) and $\Lambda^{\prime}$ of respective ranks $m_{1}$ and $m^{\prime}$, corresponding to subconnections $\left(V_{1}, \nabla_{1}\right)$ and $\left(V^{\prime}, \nabla^{\prime}\right)$ of $(V, \nabla)$ and such that $\Lambda_{K}(\nabla)=\Lambda_{1} \oplus \Lambda^{\prime}$. Then we get

$$
\Lambda_{L}(\nabla)=\left(\Lambda_{K}(\nabla)\right)_{L}(\nabla)=\left(\Lambda_{1}\right)_{L} \oplus\left(\Lambda^{\prime}\right)_{L} .
$$

The set of determinant factors of $\nabla$ is the disjoint reunion of the sets of determinant factors of $\nabla_{1}$ and $\nabla^{\prime}$; note that all determinant factors of $\nabla_{1}$ have valuation $-\kappa$.

The connections $\nabla_{1}$ and $\nabla^{\prime}$ have strictly less distinct determinant factors than $\nabla$. The induction assumption then yields

$$
\begin{aligned}
0 \leqslant\left[\Lambda_{K}(\nabla): \Lambda_{L}(\nabla)\right] \leqslant & \frac{m_{1}\left(m_{1}-1\right)}{2} \mathfrak{p}_{\Lambda_{1}}\left(\nabla_{1}\right)-\frac{1}{2} \operatorname{irr}\left(\text { End } \nabla_{1}\right) \\
& +\frac{m^{\prime}\left(m^{\prime}-1\right)}{2} \mathfrak{p}_{\Lambda^{\prime}}\left(\nabla^{\prime}\right)-\frac{1}{2} \operatorname{irr}\left(\text { End } \nabla^{\prime}\right) .
\end{aligned}
$$

According to the definition of the Katz lattice,

$$
\mathfrak{p}_{\Lambda_{K}(\nabla)}(\nabla)=\kappa=\max \left(\mathfrak{p}_{\Lambda_{1}}\left(\nabla_{1}\right), \mathfrak{p}_{\Lambda^{\prime}}\left(\nabla^{\prime}\right)\right)
$$

holds. Therefore,

$$
\begin{aligned}
0 \leqslant\left[\Lambda_{K}(\nabla): \Lambda_{L}(\nabla)\right] \leqslant & \left(\frac{m_{1}\left(m_{1}-1\right)}{2}+\frac{m^{\prime}\left(m^{\prime}-1\right)}{2}\right) \kappa \\
& -\frac{1}{2} \operatorname{irr}\left(\text { End } \nabla_{1}\right)-\frac{1}{2} \operatorname{irr}\left(\text { End } \nabla^{\prime}\right) .
\end{aligned}
$$

After Lemma 5.2, we have

$$
\frac{1}{2}\left(m_{1}\left(m_{1}-1\right)\right)+\frac{1}{2}\left(m^{\prime}\left(m^{\prime}-1\right)\right)=\frac{1}{2}(n(n-1))-m_{1} m^{\prime} .
$$

Hence, we get

$$
\begin{aligned}
0 \leqslant\left[\Lambda_{K}(\nabla): \Lambda_{L}(\nabla)\right] \leqslant & \frac{n(n-1)}{2} \kappa-m_{1} m^{\prime} \kappa \\
& -\frac{1}{2} \operatorname{irr}\left(\text { End } \nabla_{1}\right)-\frac{1}{2} \operatorname{irr}\left(\text { End } \nabla^{\prime}\right) .
\end{aligned}
$$




\section{ON FUCHS' RELATION FOR LINEAR DIFFERENTIAL SYSTEMS}

According to the assumption, the difference between a determinant factor $Q_{i}$ of $\nabla_{1}$ and a determinant factor $Q_{j}$ of $\nabla^{\prime}$ has valuation $v\left(Q_{i}-Q_{j}\right)=-\kappa$. Thus

$$
m_{1} m^{\prime} \kappa+\frac{1}{2} \operatorname{irr}\left(\operatorname{End} \nabla_{1}\right)+\frac{1}{2} \operatorname{irr}\left(\text { End } \nabla^{\prime}\right)=\frac{1}{2} \operatorname{irr}(\text { End } \nabla) .
$$

Hence, the following inequalities hold:

$$
0 \leqslant\left[\Lambda_{K}(\nabla): \Lambda_{L}(\nabla)\right] \leqslant \frac{n(n-1)}{2} \kappa-\frac{1}{2} \operatorname{irr}(\text { End } \nabla) .
$$

Relation (11) finally yields

$$
\begin{aligned}
\mathfrak{p}-\kappa \leqslant\left[\Lambda: \Lambda_{L}(\nabla)\right] & \leqslant \frac{n(n-1)}{2}(\mathfrak{p}-\kappa)+\frac{n(n-1)}{2} \kappa-\frac{1}{2} \operatorname{irr}(\text { End } \nabla) \\
& \leqslant \frac{n(n-1)}{2} \mathfrak{p}-\frac{1}{2} \operatorname{irr}(\text { End } \nabla) .
\end{aligned}
$$

b) The map $\bar{\nabla}^{\Lambda_{K}(\nabla)}$ has only one eigenvalue, which is non-zero according to condition iii of Lemma 4.6. All the attached eigenvalues (and thus all determinant factors) are equivalent up to order 0 . Let $k$ be the largest integer such that the $\varphi_{i}$ are all equivalent up to order $k$. Let us call $P \in(1 / z) \mathbb{C}[1 / z]$ the polynomial of degree $-k$ that is equivalent to all $\varphi_{i}$ up to order $k$, and consider the connection $\nabla^{\prime}=\nabla-P \mathrm{id}_{V} \otimes d z / z$. The connection $\nabla^{\prime}$ satisfies the condition a, because its determinant factors are not all equivalent. The Katz rank $\kappa^{\prime}$ of the connection $\nabla^{\prime}$ satisfies $\kappa^{\prime}<\kappa$, thus the lattice $\Lambda_{K}=\Lambda_{K}(\nabla)$ does not have minimal Poincaré rank for $\nabla^{\prime}$. Consider the Katz lattice $\Lambda_{K^{2}}=\left(\Lambda_{K}\right)_{K}\left(\nabla^{\prime}\right)$. According to Corollary 4.1, the corresponding index then satisfies

$$
\kappa-\kappa^{\prime} \leqslant\left[\Lambda_{K}(\nabla): \Lambda_{K^{2}}\right] \leqslant \frac{n(n-1)}{2}\left(\kappa-\kappa^{\prime}\right) .
$$

We then consider the Levelt lattice $\left(\Lambda_{K^{2}}\right)_{L}\left(\nabla^{\prime}\right)$ of the lattice $\Lambda_{K^{2}}$ for the connection $\nabla^{\prime}$. By means of Lemmas 4.7 and 4.2 we get

$$
\begin{aligned}
\left(\Lambda_{K^{2}}\right)_{L}\left(\nabla^{\prime}\right) & =\left(\left(\Lambda_{K}\right)_{K}\left(\nabla^{\prime}\right)\right)_{L}\left(\nabla^{\prime}\right)=\left(\Lambda_{K}\right)_{L}\left(\nabla^{\prime}\right) \\
& =\left(\Lambda_{K}\right)_{L}\left(\nabla-P \operatorname{id}_{V} \otimes \frac{d z}{z}\right) \\
& =\left(\Lambda_{K}\right)_{L}(\nabla) \\
& =\Lambda_{L}(\nabla) .
\end{aligned}
$$

Accordingly, the index $\left[\Lambda_{K^{2}}:\left(\Lambda_{K^{2}}\right)_{L}\left(\nabla^{\prime}\right)\right]$ satisfies

$$
0 \leqslant\left[\Lambda_{K^{2}}:\left(\Lambda_{K^{2}}\right)_{L}\left(\nabla^{\prime}\right)\right] \leqslant \frac{n(n-1)}{2} \kappa^{\prime}-\frac{1}{2} \operatorname{irr}\left(\text { End } \nabla^{\prime}\right) .
$$

Only differences between determinant factors occur in the Malgrange irregularity; hence we have that $\operatorname{irr}\left(\right.$ End $\left.\nabla^{\prime}\right)=\operatorname{irr}($ End $\nabla)$. Thus,

$$
\begin{aligned}
0 \leqslant\left[\Lambda_{K}(\nabla): \Lambda_{L}(\nabla)\right] & =\left[\Lambda_{K}(\nabla): \Lambda_{K^{2}}\right]+\left[\Lambda_{K^{2}}: \Lambda_{L}(\nabla)\right] \\
& \leqslant \frac{n(n-1)}{2}\left(\kappa-\kappa^{\prime}\right)+\frac{n(n-1)}{2} \kappa^{\prime}-\frac{1}{2} \operatorname{irr}\left(\text { End } \nabla^{\prime}\right) \\
& \leqslant \frac{n(n-1)}{2} \kappa-\frac{1}{2} \operatorname{irr}(\text { End } \nabla),
\end{aligned}
$$

so our induction is complete. Relation (11) then yields

$$
\begin{aligned}
\mathfrak{p}-\kappa \leqslant\left[\Lambda: \Lambda_{L}(\nabla)\right] & \leqslant \frac{n(n-1)}{2}(\mathfrak{p}-\kappa)+\frac{n(n-1)}{2} \kappa-\frac{1}{2} \operatorname{irr}(\text { End } \nabla) \\
& \leqslant \frac{n(n-1)}{2} \mathfrak{p}-\frac{1}{2} \operatorname{irr}(\text { End } \nabla) .
\end{aligned}
$$




\section{E. Corel}

\subsection{Fuchs' inequalities}

Proposition 5.2. Let $(V, \nabla)$ be a $K$-vector space endowed with a connection, and $\Lambda$ be a lattice of $V$. Denote with $\mathfrak{p}=\mathfrak{p}_{\Lambda}(\nabla)$ the Poincaré rank of the connection $\nabla$ on the lattice $\Lambda$, with $\wedge^{n} \mathfrak{p}$ the Poincaré rank of $\Lambda^{n} \nabla$ on $\Lambda^{n} \Lambda$ and with $\tau_{\Lambda}(\nabla)$ the trace of the residue of $\nabla$ on $\Lambda$. Then the sum of all exponents $e_{1}, \ldots, e_{n}$ of the connection $\nabla$ on the lattice $\Lambda$ satisfies

$$
\tau_{\Lambda}(\nabla)-\frac{n(n-1)}{2} \mathfrak{p} \leqslant \sum_{i=1}^{n} e_{i}-\frac{1}{2} \operatorname{irr}(\text { End } \nabla) \leqslant \tau_{\Lambda}(\nabla)-\mathfrak{p}+\wedge^{n} \mathfrak{p} .
$$

Proof. Assume first that the connection is unramified. The $n$ exponents $e_{i}$ are equal to the eigenvalues of the residue of the associated regular connection $\nabla^{r}$ on the Levelt lattice of $\Lambda$. By Lemma 2.4 one has

$$
\sum_{i=1}^{n} e_{i}=\tau_{\Lambda_{L}}\left(\nabla^{r}\right)=\tau_{\Lambda}\left(\nabla^{r}\right)-\left[\Lambda: \Lambda_{L}(\nabla)\right] .
$$

Since $\bigwedge^{n} \nabla=\bigwedge^{n} \nabla^{r}+\operatorname{Tr} \omega$ is the canonical decomposition of $\bigwedge^{n} \nabla$, the relation

$$
\tau_{\Lambda}\left(\nabla^{r}\right)=\tau_{\Lambda}(\nabla)-\underset{0}{\operatorname{Res}} \operatorname{Tr} \frac{1}{z} \varphi=\tau_{\Lambda}(\nabla)
$$

holds, because $\operatorname{Tr}(1 / z) \varphi \subset\left(1 / z^{2}\right) \mathbb{C}[1 / z]$. Accordingly, the sum of exponents satisfies

$$
\sum_{i=1}^{n} e_{i}=\tau_{\Lambda}(\nabla)-\left[\Lambda: \Lambda_{L}(\nabla)\right]
$$

Denote with $\kappa$ the Katz rank of $\nabla$. After Proposition 5.1, we get

$$
\tau_{\Lambda}(\nabla)-\frac{n(n-1)}{2} \mathfrak{p} \leqslant \sum_{i=1}^{n} e_{i}-\frac{1}{2} \operatorname{irr}(\text { End } \nabla) \leqslant \tau_{\Lambda}(\nabla)-\mathfrak{p}+\kappa-\frac{1}{2} \operatorname{irr}(\text { End } \nabla) .
$$

Let $\varphi_{1}, \ldots, \varphi_{n}$ be the attached eigenvalues of $\nabla$, counted without respect to their multiplicities, and assume that they are arranged by increasing valuation. Then $\kappa=-v\left(\varphi_{1}\right)$ and

$$
\operatorname{irr}(\text { End } \nabla)=-\sum_{1 \leqslant i, j \leqslant n} \min \left(v\left(\varphi_{i}-\varphi_{j}\right), 0\right)
$$

hold. The sum $\varphi_{1}+\cdots+\varphi_{n}=\operatorname{Tr} \varphi$ is equal to the only eigenvalue attached to the connection $\bigwedge^{n} \nabla$. The space $\bigwedge^{n} V$ has dimension 1 , and its Poincaré rank is

$$
\wedge^{n} \mathfrak{p}=\sup \left(0,-v\left(\varphi_{1}+\cdots+\varphi_{n}\right)\right) .
$$

Hence, we have

$$
\wedge^{n} \mathfrak{p} \leqslant \kappa \leqslant \mathfrak{p} .
$$

If there exists $i<j$ such that the equality $-v\left(\varphi_{i}-\varphi_{j}\right)=-v\left(\varphi_{1}\right)$ holds, then we have $\kappa-$ $\frac{1}{2} \operatorname{irr}($ End $\nabla) \leqslant 0$. If instead $\kappa-\frac{1}{2} \operatorname{irr}($ End $\nabla)>0$ holds, then we have $v\left(\varphi_{1}\right)=\cdots=v\left(\varphi_{n}\right)=-\kappa$, and the coefficients of valuation $-\kappa$ of all the $\varphi_{i}$ are equal, whence $v\left(\varphi_{1}+\cdots+\varphi_{n}\right)=-\kappa$. Therefrom, one gets

$$
-\mathfrak{p}+\kappa-\frac{1}{2} \operatorname{irr}(\text { End } \nabla) \leqslant-\mathfrak{p}+\wedge^{n} \mathfrak{p}
$$

The statement of the proposition is then established for the unramified case.

Assume now that $\nabla$ is ramified of order $p$. Let us use the notations of $\S 3.1$. The field $H$ is here assumed to be endowed with its natural $\zeta$-adic valuation $w$, and the invariants of $\nabla_{H}$ are defined with respect to the uniformizing parameter $\zeta$. According to Proposition 5.1 and the proof of the 


\section{ON FUCHS' RELATION FOR LINEAR DIFFERENTIAL SYSTEMS}

unramified case just given, the following inequalities hold:

$$
\begin{aligned}
\left(\mathfrak{p}_{\zeta}\right)_{\Lambda_{H}}\left(\nabla_{H}\right)-\kappa_{\zeta}\left(\nabla_{H}\right)+\frac{1}{2} \operatorname{irr}_{\zeta}\left(\text { End } \nabla_{H}\right) & \leqslant\left[\Lambda_{H}: \Lambda_{L}(\nabla)\right]_{\zeta}+\frac{1}{2} \operatorname{irr}_{\zeta}\left(\text { End } \nabla_{H}\right) \leqslant \ldots \\
& \leqslant \frac{n(n-1)}{2}\left(\mathfrak{p}_{\zeta}\right)_{\Lambda_{H}}\left(\nabla_{H}\right) .
\end{aligned}
$$

With $\left[\Lambda_{H}: \Lambda_{L}(\nabla)\right]_{\zeta}$ we denote the index as calculated in the ring $\mathbb{C}[[\zeta]]$. Recall from $\S 3.1$ that $\left(\mathfrak{p}_{\zeta}\right)_{\Lambda_{H}}\left(\nabla_{H}\right)=p \mathfrak{p}_{\Lambda}(\nabla)$. One easily sees that the same holds for all the occurring invariants:

$$
\begin{gathered}
\kappa_{\zeta}\left(\nabla_{H}\right)=p \kappa(\nabla), \quad \operatorname{irr}_{\zeta}\left(\text { End } \nabla_{H}\right)=p \operatorname{irr}(\text { End } \nabla), \\
\left(\tau_{\zeta}\right)_{\Lambda_{H}}\left(\left(\nabla^{r}\right)_{H}\right)=p \tau_{\Lambda}\left(\nabla^{r}\right), \quad\left[\Lambda_{H}: \Lambda_{L}(\nabla)\right]_{\zeta}=p\left[\Lambda: \Lambda_{L}(\nabla)\right] .
\end{gathered}
$$

The definition of the exponents in the ramified case yields

$$
\begin{aligned}
\sum_{i=1}^{n} e_{i} & =\operatorname{Tr} \operatorname{Res}_{\Lambda_{L}(\nabla)}^{c} \nabla_{H}=\frac{1}{p} \operatorname{Tr}\left(\operatorname{Res}_{\zeta}\right)_{\Lambda_{L}(\nabla)} \nabla_{H} \\
& =\frac{1}{p}\left(\left(\tau_{\zeta}\right)_{\Lambda_{H}}\left(\left(\nabla^{r}\right)_{H}\right)-\left[\Lambda: \Lambda_{L}(\nabla)\right]_{\zeta}\right) .
\end{aligned}
$$

Replacing in the expression above finishes the proof.

Let us now consider the field $\mathbb{C}(z)$ of rational fractions, endowed at all points $a \in \mathbb{P}^{1}(\mathbb{C})$ with the local valuation map $v_{a}$. Denote with $v_{a} A=\min _{1 \leqslant i, j \leqslant n} v_{a} A_{i j}$ the order at $a$ of a matrix $A$, and with $\operatorname{Res}_{z=a} f$ the residue of a function $f(z)$ at the point $z=a$. At $s \in \mathbb{P}^{1}(\mathbb{C})$, the former local definitions make sense by means of the change of local coordinate $t=z-s$ if $s \in \mathbb{C}$ and $t=1 / z$ if $s=\infty$. We denote the Poincaré rank at $s$ with $\mathfrak{p}_{s}$.

Definition 18. If the matrix $A$ has coefficients in $\mathbb{C}(z)$, we call height of the system the integer

$$
h(A)=\sum_{a \in \mathbb{P}^{1}(\mathbb{C})} \sup \left(0,-v_{a} A d z-1\right) .
$$

Theorem 4 (Fuchs' inequalities). Let $d X / d z=A X$ be a meromorphic differential system on $\mathbb{P}^{1}(\mathbb{C})$. The exponents $e_{1}^{s}, \ldots, e_{n}^{s}$ attached to this system at all points $s \in \mathbb{P}^{1}(\mathbb{C})$ satisfy

$$
-\frac{n(n-1)}{2} h(A) \leqslant \sum_{s \in \mathbb{P}^{1}(\mathbb{C})}\left(\sum_{i=1}^{n} e_{i}^{s}-\frac{1}{2} \operatorname{irr}_{s}(\text { End } \nabla)\right) \leqslant-h(A)+h(\operatorname{Tr} A)
$$

and

$$
\sum_{s \in \mathbb{P}^{1}(\mathbb{C})} \sum_{i=1}^{n} e_{i}^{s} \leqslant 0
$$

Proof. We return to $z=0$ by a change of local coordinate. The system $d X / d z=A X$ defines a connection $\nabla$ on $K^{n}$. Attach to $\mathcal{O}^{n}$ its Levelt lattice $\left(\mathcal{O}^{n}\right)_{L}$. According to Proposition 5.2, one has the following local relation:

$$
\begin{aligned}
\operatorname{Res}_{t=0} \operatorname{Tr} A-\frac{n(n-1)}{2}\left(\mathfrak{p}_{0}\right)_{\mathcal{O}^{n}}(\nabla) & \leqslant \sum_{i=1}^{n} e_{i}^{0}-\frac{1}{2} \operatorname{irr}_{0}(\text { End } \nabla) \leqslant \ldots \\
& \leqslant \operatorname{Res}_{t=0} \operatorname{Tr} A-\left(\mathfrak{p}_{0}\right)_{\mathcal{O}^{n}}(\nabla)+\left(\mathfrak{p}_{0}\right)_{\bigwedge^{n} \mathcal{O}^{n}}\left(\bigwedge^{n} \nabla\right) .
\end{aligned}
$$




\section{E. Corel}

On the other hand, according to relation (12), one has

$$
\sum_{i=1}^{n} e_{i}^{0} \leqslant \operatorname{Res}_{t=0} \operatorname{Tr} A-\left[\mathcal{O}^{n}:\left(\mathcal{O}^{n}\right)_{L}\right]
$$

We know that $\left(\mathfrak{p}_{0}\right) \bigwedge^{n} \mathcal{O}^{n}\left(\bigwedge^{n} \nabla\right)=\sup \left(0,-v_{0}(\operatorname{Tr} A d z)-1\right)$. Adding together these inequalities at every singularity one gets

$$
\begin{aligned}
& \sum_{s \in \mathbb{P}^{1}(\mathbb{C})} \operatorname{Res}_{t=s} \operatorname{Tr} A-\frac{n(n-1)}{2} \sum_{s \in \mathbb{P}^{1}(\mathbb{C})} \mathfrak{p}_{s} \leqslant \sum_{s \in \mathbb{P}^{1}(\mathbb{C})} \sum_{i=1}^{n} e_{i}^{s}-\frac{1}{2} \operatorname{irr}_{s}(\text { End } \nabla) \leqslant \ldots \\
& \leqslant \sum_{s \in \mathbb{P}^{1}(\mathbb{C})} \operatorname{Res}_{t=s} \operatorname{Tr} A-\sum_{s \in \mathbb{P}^{1}(\mathbb{C})} \mathfrak{p}_{s}+\sum_{s \in \mathbb{P}^{1}(\mathbb{C})} \mathfrak{p}_{s}(\operatorname{Tr} A)
\end{aligned}
$$

and

$$
\sum_{s \in \mathbb{P}^{1}(\mathbb{C})} \sum_{i=1}^{n} e_{i}^{s} \leqslant \sum_{s \in \mathbb{P}^{1}(\mathbb{C})} \operatorname{Res}_{t=s} \operatorname{Tr} A .
$$

Since $\sum_{s \in \mathbb{P}^{1}(\mathbb{C})} \mathfrak{p}_{s}=h(A)$, both results follow now from the residue theorem.

Let $A \in \mathrm{M}_{n}(\mathbb{C}(z))$ be a matrix of rational functions having poles in the set $S=\left\{s_{1}, \ldots, s_{p}\right\} \subset$ $\mathbb{P}^{1}(\mathbb{C})$. For every $s \in S$, denote its Poincaré rank with $\mathfrak{p}_{s}=\max \left(0,-v_{s} A d z-1\right)$, and its polar matrix with the matrix

$$
\begin{gathered}
\bar{A}_{s}=\lim _{z \rightarrow s}(z-s)^{\mathfrak{p}_{s}+1} A(z) \quad \text { if } s \neq \infty, \\
\bar{A}_{\infty}=-\lim _{t \rightarrow 0} t^{\mathfrak{p}_{\infty}-1} A\left(\frac{1}{t}\right) \quad \text { for } s=\infty .
\end{gathered}
$$

We say that $s \in S$ is a singularity of first kind if $\mathfrak{p}_{s}=0$, and of second kind if $\mathfrak{p}_{s}>0$.

Definition 19. We say that the system $d X / d z=A X$ is generic if for every singularity $s$ of the second kind of $A$ the polar matrix $\bar{A}_{s}$ has $n$ distinct eigenvalues.

Corollary 5.1. Let $d X / d z=A X$ be a generic system over $\mathbb{P}^{1}(\mathbb{C})$. The sum of its exponents $e_{1}^{s}, \ldots, e_{n}^{s}$ at all points $s \in \mathbb{P}^{1}(\mathbb{C})$ satisfies

$$
\sum_{s \in \mathbb{P}^{1}(\mathbb{C})} \sum_{i=1}^{n} e_{i}^{s}=0
$$

Proof. Let $s$ be a singularity of the second kind. Let $\varphi_{1}^{s}, \ldots, \varphi_{n}^{s}$ be the determinant factors attached to the system at $s$. Since the system is generic, one has

$$
v_{s}\left(\varphi_{i}^{s}\right)=v_{s}\left(\varphi_{i}^{s}-\varphi_{j}^{s}\right)=-\mathfrak{p}_{s}
$$

for all $1 \leqslant i \neq j \leqslant n$. The local Malgrange irregularity index at $s$ is then equal to

$$
\operatorname{irr}_{s}(\text { End } \nabla)=n(n-1) \mathfrak{p}_{s} .
$$

If $s$ is of the first kind, then it is a regular singularity and the same relation is satisfied. Hence one has

$$
\sum_{s \in \mathbb{P}^{1}(\mathbb{C})}\left(\sum_{i=1}^{n} e_{i}^{s}-\frac{1}{2} \operatorname{irr}_{s}(\text { End } \nabla)\right)=\sum_{s \in \mathbb{P}^{1}(\mathbb{C})} \sum_{i=1}^{n} e_{i}^{s}-\frac{n(n-1)}{2} h(A) .
$$




\section{ON FUCHS' RELATION FOR LINEAR DIFFERENTIAL SYSTEMS}

According to relation (13) of Theorem 4 we get

$$
\sum_{s \in \mathbb{P}^{1}(\mathbb{C})} \sum_{i=1}^{n} e_{i}^{s} \geqslant 0 .
$$

Relation (14) of Theorem 4 then yields the result.

\section{Appendix}

In this appendix, we describe the algorithm whose existence was mentioned in Remark 8 . The general idea is to compute an $\mathcal{O}$-basis of the lattice $\Lambda_{k}(\nabla)$ by using its description in terms of the saturated Gérard-Levelt lattice given in Corollary 4.3. Note that if the connection $\nabla$ has matrix representation $A=\operatorname{Mat}\left(\nabla_{\theta},(e)\right)$ in an $\mathcal{O}$-basis $(e)$ of $\Lambda$, then its saturated Gérard-Levelt lattice $\mathcal{F}_{z^{k} \theta}^{n-1}(\Lambda)$ is spanned by the columns of the $n \times n^{2}$ matrix

$$
\mathcal{M}_{k}(\nabla,(e))=\mathcal{M}\left(z^{k} A\right)=\left(I \mathcal{A} \mathcal{A}_{2} \ldots \mathcal{A}_{n-1}\right),
$$

where

$$
\begin{aligned}
\mathcal{A}_{0} & =I \\
\mathcal{A}_{t+1} & =z^{k} \theta \mathcal{A}_{t}+z^{k} A \mathcal{A}_{t} \quad \text { for any } t \geqslant 0 .
\end{aligned}
$$

Since

to the differential system

$$
\Lambda_{k}(\nabla)=\mathcal{F}_{z^{k} \theta}^{n-1}\left(\Lambda^{*}\right)^{*}
$$

$$
\frac{d X}{d z}=A X
$$

we attach $n$ column vectors spanning the same $\mathcal{O}$-module as the $n^{2}$ columns of the matrix $\mathcal{M}_{\mathcal{F}}\left(-f^{\mathrm{t}} A\right)$ defined from the dual system $d X / d z=-{ }^{t} A X$, for some well-chosen $f \in K$.

The following section describes the tools to perform this procedure.

\section{Hermite normal form}

Let $E$ be a euclidean ring, and let $m, n \in \mathbb{N}$ be two integers. Denote with $\mathrm{M}_{n \times m}(E)$ the algebra of $n \times m$ matrices with coefficients in $E$. Assume that $n \leqslant m$.

Theorem 5 (Hermite normal form). Let $M=\left(M_{i j}\right) \in \mathrm{M}_{n \times m}(E)$ an $n \times m$ matrix with coefficients in $E$. Then there exists a matrix $U \in \mathrm{GL}_{m}(E)$ such that $M U$ has the following form:

$$
M U=\left(\begin{array}{ccccccc}
0 & \ldots & 0 & m_{11} & m_{12} & \ldots & m_{1 n} \\
0 & \ldots & 0 & 0 & m_{22} & \ldots & m_{2 n} \\
\vdots & & \vdots & \vdots & & \ddots & \\
0 & \ldots & 0 & 0 & 0 & & m_{n n}
\end{array}\right) .
$$

Since $U \in \mathrm{GL}_{m}(E)$, the $n$ last columns of $M U$ span the same $E$-module as the $m$ columns of $M$.

This theorem holds for the ring of polynomials $\mathbb{C}[z]$ (see e.g. [Coh91, p. 69], or [Roc93, ch. VI]). One can moreover assume in this case that the polynomials $m_{i i}$ have leading coefficient for all $i=1, \ldots, n$ and that $d^{\circ} m_{i i}>d^{\circ} m_{i j}$ for all $j>i$.

\section{Description of the algorithm}

Let us consider a differential system $d X / d z=A X$ with coefficients in the field $K=\mathbb{C}(z)$. Let $V=K^{n}$, and define $\nabla$ as the connection such that $\nabla_{d / d z}$ has matrix $A$ in the canonical basis of $V$. 


\section{E. Corel}

For every pole $z=a_{i}$ of the matrix $A$, the localized ring $R_{i}=\mathbb{C}[z]_{\left(z-a_{i}\right)}$ of $\mathbb{C}[z]$ at the principal ideal $\left(z-a_{i}\right)$ is the valuation subring of $K$ for the $\left(z-a_{i}\right)$-adic valuation $v_{i}$. Embed then $K$ in the field $\mathbb{C}\left(\left(z-a_{i}\right)\right)$ of all formal series in $\left(z-a_{i}\right)$ with coefficients in $\mathbb{C}$. Denote with $m_{i}$ the order of singularity of the pole $a_{i}$. Finally set $\Lambda_{i}=\left(R_{i}\right)^{n}$ and denote with $\Lambda_{i}^{*}$ its dual.

Theorem 2 of Gérard and Levelt [GL73] ensures that

$$
\nabla_{\left(z-a_{i}\right)^{m_{i} d / d z}}^{*}\left(\mathcal{F}_{\left(z-a_{i}\right)^{m_{i} d / d z}}^{n-1}\left(\Lambda_{i}^{*}\right)\right) \subset \mathcal{F}_{\left(z-a_{i}\right)^{m_{i} d / d z}}^{n-1}\left(\Lambda_{i}^{*}\right) .
$$

The lattice $\mathcal{F}_{\left(z-a_{i}\right)^{m_{i} d / d z}}^{n-1}\left(\Lambda_{i}^{*}\right)$ is the Gérard-Levelt saturated lattice of $\Lambda_{i}^{*}$ of order $m_{i}$ with respect to the uniformizing parameter $t_{i}=z-a_{i}$.

This process can be simultaneously performed at every finite singularity of the system. Let $S=\left\{a_{1}, \ldots, a_{p}\right\}$ be the set of poles of the matrix $A$ contained in $\mathbb{C}$. Set $f=\left(z-a_{1}\right)^{m_{1}}$ $\left(z-a_{2}\right)^{m_{2}} \cdots\left(z-a_{p}\right)^{m_{p}}$.

Proposition A.1. Let $\vartheta$ be the derivation $\vartheta=f d / d z$ of $K$. Then the following hold.

1) The lattice $\mathcal{F}_{\vartheta}^{n-1}\left(\Lambda_{i}^{*}\right)^{*}$ is the largest $\left(z-a_{i}\right)^{m_{i}} \nabla_{d / d z}$-stable sublattice of $\Lambda_{i}$ for any $i=1, \ldots, p$.

2) There exists a $K$-basis $(e)$ of $V$ such that the lattice $\mathcal{F}_{\vartheta}^{n-1}\left(\Lambda_{i}^{*}\right)^{*}$ is spanned over $R_{i}$ by $(e)$ for any $i=1, \ldots, p$.

Proof. The derivation $\vartheta$ satisfies $\vartheta=\prod_{j \neq i}\left(z-a_{j}\right)\left(z-a_{i}\right) d / d z$ for all $i=1, \ldots, p$. Since $\prod_{j \neq i}\left(z-a_{j}\right)$ is invertible in $R_{i}$ for any $i=1, \ldots, p$, one has $\mathcal{F}_{\vartheta}^{n-1}(M)=\mathcal{F}_{\left(z-a_{i}\right) d / d z}^{n-1}(M)$ for any $R_{i}$-lattice $M$ of $V$. This result also clearly holds for the dual. Since $\mathcal{F}_{\vartheta}^{n-1}\left(\Lambda_{i}^{*}\right)$ is the smallest $\left(z-a_{i}\right)^{m_{i}} \nabla_{d / d z}$-stable lattice containing $\Lambda_{i}^{*}$, its dual lattice $\mathcal{F}_{\vartheta}^{n-1}\left(\Lambda_{i}^{*}\right)^{*}$ is the largest $\left(z-a_{i}\right)^{m_{i}} \nabla_{d / d z}$-stable sublattice of $\Lambda_{i}$. Thus part 1 is proved.

The lattice $\mathcal{F}_{\vartheta}^{n-1}\left(\Lambda_{i}^{*}\right)$ is spanned in the canonical basis of $K^{n}$ by the columns of

$$
\mathcal{M}\left(-f^{\mathrm{t}} A\right)=\left(I \mathcal{A} \mathcal{A}_{2} \ldots \mathcal{A}_{n-1}\right),
$$

where

$$
\begin{aligned}
\mathcal{A}_{0} & =I \\
\mathcal{A}_{k+1} & =\vartheta \mathcal{A}_{k}-f^{\mathrm{t}} A \mathcal{A}_{k}, \quad \text { for any } k \geqslant 0,
\end{aligned}
$$

for any $i=1, \ldots, p$. Since the columns of $\mathcal{M}\left(-f^{\mathrm{t}} A\right)$ are independent of $i$, the statement for part 2 follows.

The next step is to find the basis $(e)$ of Proposition A.1. In order to perform Hermite's reduction on the matrix $\mathcal{M}\left(-f^{\mathrm{t}} A\right)$ whose coefficients belong to $\mathbb{C}(z)$, consider $q \in \mathbb{C}[z]$ such that the matrix $M=q \mathcal{M}\left(-f^{\mathrm{t}} A\right)$ is polynomial and of zero valuation. After Theorem 5 , there exists $U \in \mathrm{GL}_{n^{2}}(\mathbb{C}[z])$ such that $M U$ is of the form (A5). Let us denote with $\tilde{M}$ the upper triangular matrix consisting of the last $n$ columns of $M U$ :

$$
\tilde{M}=\left(\begin{array}{ccc}
m_{11} & \ldots & m_{1 n} \\
\vdots & \ddots & \\
0 & & m_{n n}
\end{array}\right)
$$

The block matrix consisting of the first $n$ columns of $M$ is $q I$, so $M$ has rank $n$ over $K$. Hence, according to Theorem 5 , the matrix $\tilde{M}$ has also rank $n$ over $K$.

Proposition A.2. If the system (A4) has only regular singularities over $\mathbb{P}^{1}(\mathbb{C})$, the system

$$
\frac{d X}{d z}=A_{\left[{ }^{\mathrm{t}} \tilde{M}^{-1}\right]} X
$$

has only simple poles over $\mathbb{C}$, and these poles belong to $S=\left\{a_{1}, \ldots, a_{p}\right\}$. Moreover, the eigenvalues of $\operatorname{Res}_{z=s} A_{\left[{ }^{t} \tilde{M}^{-1}\right]}$ are the exponents of the system $d X / d z=A X$ at any $s \in \mathbb{C}$. 


\section{ON FUCHS' RELATION FOR LINEAR DIFFERENTIAL SYSTEMS}

Proof. Let us denote with $(u)$ the canonical basis of $V=K^{n}$. The matrix $\tilde{M}$ is the gauge matrix from the dual basis $\left(u^{*}\right)$ of $V^{*}$ to a basis $(\alpha)$ of the saturated dual lattice $\mathcal{F}_{\vartheta}^{n-1}\left(\Lambda_{i}^{*}\right)$. This basis spans

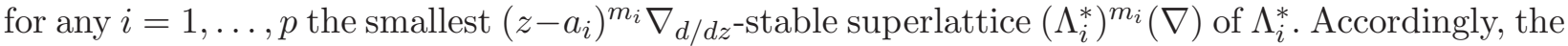
matrix ${ }^{\mathrm{t}} \tilde{M}^{-1}$ is a gauge matrix from $(u)$ to the basis $\left(\alpha^{*}\right)$ which spans the largest $\left(z-a_{i}\right)^{m_{i}} \nabla_{d / d z^{-}}$ stable sublattice of $\Lambda_{i}$ that we denoted with $\left(\Lambda_{i}\right)_{m_{i}}$ in $\S 2.3$. The basis $\left(\alpha^{*}\right)$ then satisfies the conditions of the basis $(e)$ of Proposition A.1.

The matrix $U$ belongs to $\mathrm{GL}_{n}\left(R_{i}\right)$ for any $i=1, \ldots, p$. Denote with $H(s)$ evaluation at any point $z=s \in \mathbb{C}$ of a matrix function $H \in \mathrm{M}_{n \times m}(\mathbb{C}[z])$. For $s \notin S$, we have $q(s) \neq 0$, so $M(s) \in \mathrm{M}_{n \times n^{2}}(\mathbb{C})$ has rank $n$ over $\mathbb{C}$. According to Theorem 5, the matrix $U(s)$ has rank $n^{2}$ over $\mathbb{C}$. Hence the matrix $\tilde{M}(s)$ has also rank $n$ over $\mathbb{C}$, and thus the polynomial $m_{11} m_{22} \cdots m_{n n}$ has no zero outside of $S$. Therefore, $A_{\left[{ }^{t} \tilde{M}^{-1}\right]}$ does not bring any apparent singularity outside of $S$.

If $s \in \mathbb{C}$ is a regular point for the system (A4), it is also regular for the system $d X / d z=$ $A_{\left[{ }^{t} \tilde{M}^{-1}\right]} X$. At a regular point, the exponents are all zero, and indeed one has $\operatorname{Res}_{z=s} A_{\left[{ }^{t} \tilde{M}^{-1}\right]}=0$. The assumption that the system has only regular singularities over $\mathbb{P}^{1}(\mathbb{C})$ means that $m_{i}=0$ for all $i=1, \ldots, p$. Therefore, the lattice $\left(\Lambda_{i}\right)_{m_{i}}$ spanned by $(e)$ is the regular Levelt lattice of $\Lambda_{i}$ for all $i=1, \ldots, p$ such that $a_{i} \in \mathbb{C}$, hence the eigenvalues of $\operatorname{Res}_{z=a_{i}} A_{\left[{ }^{t} \tilde{M}^{-1}\right]}$ are the exponents of the system $d X / d z=A X$ at $a_{i}$.

\section{REFERENCES}

AB94 D. V. Anosov and A. A. Bolibrukh, The Riemann-Hilbert problem, Aspects of Mathematics, vol. E22 (Vieweg \& Sohn, Braunschweig, 1994).

BV83 D. G. Babbitt and V. S. Varadarajan, Formal reduction theory of meromorphic differential equations: a group theoretic view, Pacific J. Math. 109 (1983), 1-80.

BJL79 W. Balser, W. B. Jurkat and D. A. Lutz, A general theory of invariants for meromorphic differential equations, Part I: Formal invariants, Funkcial. Ekvac. 22 (1979), 197-221.

BB85 D. Bertrand and F. Beukers, Équations différentielles linéaires et majorations de multiplicités, Ann. Sci. Écoles Norm. Sup. 18 (1985), 181-192.

BL85 D. Bertrand and G. Laumon, Appendice à exposants, vecteurs cycliques et majorations de multiplicités, C. R. Conf. Franco-Japonaise, Strasbourg, Preprint IRMA (1985).

Ber79 D. Bertrand, Travaux récents sur les points singuliers des équations différentielles linéaires, Sém. Bourbaki 538 (1979).

Ber98 D. Bertrand, On André's proof of the Siegel-Shidlovski theorem, in Colloque Franco-Japonais: Théorie des nombres transcendants (Tokyo, 1998), Sem. Math. Sci. 27 (Keio University, Yokohama, 1999), 51-63.

Bol90 A. A. Bolibrukh, The Riemann-Hilbert problem, Russian Math. Surveys 45 (1990), 1-58.

Bol95 A. A. Bolibrukh, Hilbert's twenty-first problem for linear Fuchsian systems, in Proc. Steklov Inst. Math., vol. 206(5) (Amer. Math. Soc., Providence, RI, 1995).

Bou85 N. Bourbaki, Algèbre commutative (Masson, Paris, 1985), ch. 5-7.

Coh91 H. Cohen, A course in computational algebraic number theory, Graduate Texts in Mathematics, vol. 138 (Springer, Berlin, 1991).

Cor99a E. Corel, Inégalités de Fuchs pour les systèmes différentiels réguliers, C. R. Acad. Sci. Paris 328 (1999), 983-986.

Cor99b E. Corel, Exposants, réseaux de Levelt et relations de Fuchs pour les systèmes différentiels réguliers, Thèse de Doctorat de $3^{\text {ème }}$ cycle, Univ. Louis Pasteur, Strasbourg (1999).

Cor01a E. Corel, Relations de Fuchs pour les systèmes différentiels réguliers, Bull. Soc. Math. France 129 (2001), 189-210.

Cor01b E. Corel, Relations de Fuchs pour les systèmes différentiels irréguliers, C. R. Acad. Sci. Paris 333 (2001), 297-300. 


\section{ON FUCHS' RELATION FOR LINEAR DIFFERENTIAL SYSTEMS}

Cor01c E. Corel, La relation de Fuchs pour les systèmes différentiels linéaires, Preprint no. 299, Inst. Math. Jussieu, Paris (2001).

Del70 P. Deligne, Équations différentielles à points singuliers réguliers, Lecture Notes in Mathematics, vol. 163 (Springer, Berlin, 1970).

Fab85 É. Fabry, Sur les intégrales des équations différentielles linéaires à coefficients rationnels, Thèse, Paris (1885).

GL73 R. Gérard and A. H. M. Levelt, Invariants mesurant l'irrégularité en un point singulier d'un système d'équations différentielles linéaires, Ann. Inst. Fourier (Grenoble) 23 (1973), 157-195.

Huk37 M. Hukuhara, Sur les points singuliers des équations différentielles linéaires, II, J. Fac. Sci. Hokkaido Univ. 5 (1937), 123-166.

Jur78 W. Jurkat, Meromorphe differentialgleichungen, Lecture Notes in Mathematics, vol. 637 (Springer, Berlin, 1978).

Lev61 A. H. M. Levelt, Hypergeometric functions, II, Nederl. Akad. Wetensch. Proc. Ser. A 64 (1961), 373-385.

Lev75 A. H. M. Levelt, Jordan decomposition for a class of singular differential operators, Arkiv för matematik 13 (1975), 1-27.

Lev01 A. H. M. Levelt, Appendice à l'article d'Eduardo Corel: Calcul des réseaux de Levelt, Bull. Soc. Math. France 129 (2001), 211-213.

Mal83 B. Malgrange, La classification des connexions irrégulières à une variable, Sémin. Écoles Norm. Sup. 1981-1982, partie IV, exposé no. 6 (Birkhäuser, Basel, 1983).

Man65 Y. Manin, Moduli fuchsiani, Ann. Scuola Norm. Sup. Pisa (3) 19 (1965), 113-126.

Mar96 J. Martinet, Les réseaux des espaces euclidiens (Masson, Paris, 1996).

Poo60 E. G. C. Poole, Introduction to the theory of linear differential equations (Dover, New York, 1960).

Rob80 P. Robba, Lemmes de Hensel pour les opérateurs différentiels. Applications à la réduction formelle des équations différentielles, Enseign. Math. 26 (1980), 279-311.

Roc93 F. Roch, Calcul formel et parallélisme: forme normale d'Hermite, méthodes de calcul et parallélisation, Thèse de doctorat (Math. Appl.), Inst. Polytechnique de Grenoble (1993).

Ser68 J. P. Serre, Corps locaux (Hermann, Paris, 1968).

Tur55 H. Turrittin, Convergent solutions of ordinary linear homogeneous differential equations in the neighbourhood of an irregular singular point, Acta Math. 93 (1955), 27-66.

Var91 V. S. Varadarajan, Meromorphic differential equations, Expo. Math. 9 (1991), 97-188.

Was65 W. Wasow, Asymptotic expansions for ordinary differential equations, Pure and Applied Mathematics, vol. 14 (Interscience, New York, 1965).

Eduardo Corel corel@math.jussieu.fr

Laboratoire AGAT, Université des Sciences et Technologies Lille 1, Cité Scientifique, 59655 Villeneuve d'Ascq Cedex, France

Current address: 14, rue Lepic, 75018 Paris, France 